\title{
ON LOCALLY ANALYTIC BEILINSON-BERNSTEIN LOCALIZATION AND THE CANONICAL DIMENSION
}

\author{
TOBIAS SCHMIDT
}

\begin{abstract}
Let $\mathbf{G}$ be a connected split reductive group over a $p$-adic field. In the first part of the paper we prove, under certain assumptions on $\mathbf{G}$ and the prime $p$, a localization theorem of Beilinson-Bernstein type for admissible locally analytic representations of principal congruence subgroups in the rational points of $\mathbf{G}$. In doing so we take up and extend some recent methods and results of Ardakov-Wadsley on completed universal enveloping algebras (4 $)$ to a locally analytic setting. As an application we prove, in the second part of the paper, a locally analytic version of Smith's theorem on the canonical dimension.
\end{abstract}

MSC2000: 11F85, 22E50, 16S30.

\section{Contents}

1. Introduction

2. Preliminaries on crossed products and sheaves

3. Differential operators on homogeneous spaces

4. Deformations and completions

5. Locally analytic representations

6. An application of an isomorphism of Lazard

7. Congruence kernels in semisimple $p$-adic groups

8. The equivalence of categories

9. Dimension computations

References

\section{Introduction}

Let $L / \mathbb{Q}_{p}$ be a finite field extension with ramification index $e$. Let $\mathbf{G}$ be a connected split reductive algebraic group over $L$ with group of rational points $\mathbf{G}(L)$. In a series of papers ([51, [52],554,[55]) Schneider-Teitelbaum have developed a theory of admissible locally analytic representations of the $p$-adic Lie group $\mathbf{G}(L)$ in $p$-adic locally convex vector spaces. Originally constructed for the reductive group side in the emerging $p$-adic local 
Langlands programme ([50]) this theory has found diverse other arithmetic applications, for example in the field of $p$-adic automorphic forms and $p$-adic interpolation ([20], [36]). Motivated by the theory of Harish-Chandra modules for real reductive Lie groups one may ask whether or not a good localization theory of Beilinson-Bernstein type exists for such representations. We recall that the classical Beilinson-Bernstein localization theorem ([5]) asserts an equivalence of categories between representations of a complex reductive Lie algebra with fixed central character and a category of twisted $D$-modules on the flag variety. Among other applications - notably the proof of the Kazhdan-Lusztig multiplicity conjecture - this theorem can be used to obtain a classification of the irreducible admissible smooth representations of a given real reductive Lie group.

In the first part of this paper we make a modest step in this direction and prove a localization theorem for representations of a prominent series of compact open subgroups of $\mathbf{G}(L)$, namely the principal congruence subgroups. In doing so we take up and extend some recent methods and results of Ardakov-Wadsley on completed universal enveloping algebras (4]) to a locally analytic setting. To give more details, we fix an extension of $\mathbf{G}$ to a split reductive group scheme over $o_{L}$ and denote it by the same letter. Our results are valid under three hypotheses on the geometric closed fibre $\mathbf{G}_{\bar{s}}$ of $\mathbf{G}$ which are familiar from the theory of modular Lie algebras (cf. [26]): the derived group of $\mathbf{G}_{\bar{s}}$ is (semisimple) simply connected, the prime $p$ is good for the modular Lie algebra $\operatorname{Lie}\left(\mathbf{G}_{\bar{s}}\right)$ and there exists a $\mathbf{G}_{\bar{s}}$-invariant non-degenerate bilinear form on $\operatorname{Lie}\left(\mathbf{G}_{\bar{s}}\right)$. A prominent example satisfying these conditions for all primes $p$ is the general linear group. Moreover, any almost simple and simply connected $\mathbf{G}_{\bar{s}}$ satisfies these conditions whenever $p \geq 7$ (and assuming additionally that $p$ does not divide $n+1$ in case $\mathbf{G}_{\bar{s}}$ is of type $A_{n}$ ). Assuming these hypotheses in the following, let $\mathfrak{g}$ be the $o_{L}$-Lie algebra of the group scheme $\mathbf{G}$. For any $k \geq 1$ we have the $k$-th principal congruence subgroup

$$
G:=\operatorname{ker}\left(\mathbf{G}\left(o_{L}\right) \longrightarrow \mathbf{G}\left(o_{L} / \pi_{L}^{k} o_{L}\right)\right) .
$$

We fix a $p$-adic coefficient field $K$, a finite extension of $\mathbb{Q}_{p}$. Let $U\left(\mathfrak{g}_{K}\right)$ be the universal enveloping algebra of $\mathfrak{g}_{K}=\mathfrak{g} \otimes_{\mathbb{Z}_{p}} K$ and let $\theta$ be a central character of $U\left(\mathfrak{g}_{K}\right)$. Let $\operatorname{Rep}(G)_{\theta}$ be the abelian category of admissible locally analytic $G$-representations over $K$ having infinitesimal character $\theta$. On the other hand, let $\mathbf{T}$ be a maximal split torus of $\mathbf{G}$ and $\mathbf{B} \subset \mathbf{G}$ a Borel subgroup scheme containing it. In accordance with the classical situation our localizations will live on the flag scheme

$$
X:=\mathbf{G} / \mathbf{B}
$$

of $\mathbf{G}$. Let $\mathfrak{t} \subset \mathfrak{g}$ be the Lie algebra of $\mathbf{T}$. Up to a finite extension of $K$ we may pick a weight $\lambda \in \mathfrak{t}_{K}^{*}$ that maps to $\theta$ under the classical (untwisted) Harish-Chandra mapping. Let $\rho$ be half the sum over the positive roots. Suppose $\lambda+\rho$ is dominant and regular. In this situations our main result is an equivalence of categories

$$
\mathscr{C}_{\mathcal{A}_{K}^{\lambda}} \stackrel{\cong}{\longrightarrow} \operatorname{Rep}(G)_{\theta}
$$


where $\mathcal{A}_{K}^{\lambda}$ is a certain sheaf of noncommutative $K$-algebras on $X$ and $\mathscr{C}_{\mathcal{A}_{K}^{\lambda}}$ is an explicitly given full abelian subcategory of all (left) $\mathcal{A}_{K}^{\lambda}$-modules. The latter contains, for example, all coherent modules. The functor is given essentially by the global section functor. A quasi-inverse can be made explicit using the central reduction

$$
D(G)_{\theta}:=D(G) \otimes_{Z\left(\mathfrak{g}_{K}\right), \theta} K
$$

of the locally analytic distribution algebra $D(G)$ of $G$. We remark here that the category $\operatorname{Rep}(G)_{\theta}$ is in natural duality with a full subcategory of $D(G)_{\theta}$-modules, the so-called coadmissible modules ([54]). The sheaf $\mathcal{A}_{K}^{\lambda}$ is a natural ring extension of a suitable Fréchet completion, so to speak, of the classical $\lambda$-twisted differential operators $\mathcal{D}_{K}^{\lambda}$ on the generic fibre $X_{L}$. In fact, there will be a canonical morphism $j_{*} \mathcal{D}_{K}^{\lambda} \rightarrow \mathcal{A}_{K}^{\lambda}$ inducing a commutative diagram

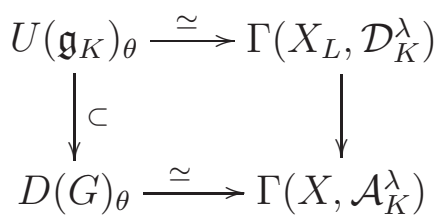

where $j: X_{L} \hookrightarrow X$ equals the inclusion of the generic fibre into $X$ and the upper isomorphism comes from [5].

As in the case of real Lie groups, the above localization theorem can be used to obtain significant information on the irreducible objects in the category $\operatorname{Rep}(G)_{\theta}$. We hope to come back to this in the future.

To sketch the construction of the sheaf $\mathcal{A}_{K}^{\lambda}$ we let $\mathbf{N}$ be the unipotent radical of $\mathbf{B}$ and put $\tilde{X}:=\mathbf{G} / \mathbf{N}$. Since $\mathbf{T}$ normalizes $\mathbf{N}$ the canonical projection

$$
\xi: \tilde{X} \longrightarrow X
$$

is a right $\mathbf{T}$-torsor for the Zariski topology on $X$. There are sheaves $\mathcal{D}_{\tilde{X}}$ and $\mathcal{D}_{X}$ of crystalline (i.e. no divided powers) differential operators on $\tilde{X}$ and $X$ respectively, familiar from the theory of arithmetic $D$-modules $([7],[8])$. We denote by

$$
\tilde{\mathcal{D}}:=\xi_{*}\left(\mathcal{D}_{\tilde{X}}\right)^{\mathbf{T}}
$$

the relative universal enveloping algebra of the torsor $\xi$ in the sense of Borho-Brylinski ([10]). Recall that if $U \subset X$ is an open affine subset trivializing $\xi$ then a choice of such a trivialization induces an isomorphism

$$
\tilde{\mathcal{D}}(U) \stackrel{\cong}{\longrightarrow} \mathcal{D}_{X}(U) \otimes_{\mathbb{Z}_{p}} U(\mathfrak{t})
$$

for the local sections of $\tilde{\mathcal{D}}$ above $U$. The homomorphisms $U(\mathfrak{t}) \rightarrow \tilde{\mathcal{D}}(U)$ for varying $U$ glue to a central embedding $U(\mathfrak{t}) \longrightarrow \tilde{\mathcal{D}}$ so that toral weights give rise to central reductions of $\tilde{\mathcal{D}}$. 
Let $\pi_{K}$ be a uniformizer of $K$ and $o_{K} \subset K$ the ring of integers. In the following we restrict to numbers $m>>0$ such that $\lambda\left(\pi_{L}^{m} \mathfrak{t}\right) \subseteq o_{K}$. For any such number $m$ we put $n:=(m-1) e+k$ and let $\widehat{\mathcal{D}_{n, K}^{\lambda}}$ be the central reduction along $\lambda$ of the $p$-adic completion (with $\pi_{K}$-inverted) of the $n$-th deformation of the sheaf $\tilde{\mathcal{D}}$ as introduced by ArdakovWadsley ([4]). Over an open affine subset $U \subset X$ trivializing $\xi$ its algebra of sections is noncanonically isomorphic to a certain $K$-Banach algebra completion of $\tilde{\mathcal{D}}_{X}(U) \otimes_{o_{L}} K$. The Banach norm in question depends on the 'deformation parameter' $n$. We remark in passing that, in loc.cit., the sheaves $\widehat{\mathcal{D}_{n, K}^{\lambda}}$ are used to establish a Beilinson-Bernstein theorem for $p$-adic completions of the universal enveloping algebra $U(\mathfrak{g})$. We make heavy use of this result. In this light our methods and results are simply a direct and straightforward extension of the corresponding ones in loc.cit.

To go further, the natural G-equivariant structure of $\mathcal{D}_{X}$ extends to $\widehat{\mathcal{D}_{n, K}^{\lambda}}$. Since the latter sheaf is supported only on the special fibre of $X$ and the latter is set-theoretically fixed by $G$ we have a group homomorphism $\sigma: G \rightarrow \operatorname{Aut}\left(\widehat{\mathcal{D}_{n, K}^{\lambda}}\right)$.

To simplify the exposition in this introduction we assume from now on $k \geq e$. We then denote by $H_{m}$ the finite group equal to the quotient of $G$ modulo its normal subgroup generated by $p^{m}$-th powers. A careful choice of a section $H_{m} \rightarrow G$ combined with results of M. Lazard on $p$-valued groups ([32]) produces from $\sigma$ a homomorphism

$$
\sigma_{m}: H_{m} \longrightarrow \operatorname{Out}\left(\widehat{\mathcal{D}_{n, K}^{\lambda}}\right)
$$

into the group of outer automorphisms of the sheaf $\widehat{\mathcal{D}_{n, K}^{\lambda}}$ as well as a 2-cocycle

$$
\tau_{m}: H_{m} \times H_{m} \longrightarrow \Gamma\left(X, \widehat{\mathcal{D}_{n, K}^{\lambda}}\right)^{\times}
$$

with respect to $\sigma_{m}$. Sheafifying the usual construction from noncommutative ring theory (43]) we obtain a crossed product sheaf

$$
\mathcal{A}_{m, K}^{\lambda}:=\widehat{\mathcal{D}_{n, K}^{\lambda}} *_{\sigma_{m}, \tau_{m}} H_{m}
$$

on $X$ for any $m>>0$. The latter is a sheaf of noncommutative associative $K$-algebras naturally containing $\widehat{\mathcal{D}_{n, K}^{\lambda}}$ as a subsheaf. The sheaves $\mathcal{A}_{m, K}^{\lambda}$ form a projective system with well-behaved transition maps and the limit

$$
\mathcal{A}_{K}^{\lambda}:={\underset{m}{m}}_{\lim _{m, K}} \mathcal{A}_{m}^{\lambda}
$$

is our promised sheaf. All sheaves $\mathcal{A}_{m, K}^{\lambda}$ are coherent and the abelian category $\mathscr{C}_{\mathcal{A}_{K}^{\lambda}}$ arises as a suitable projective limit construction involving the categories of coherent $\mathcal{A}_{m, K^{-}}^{\lambda}$ modules for all $m$. In this situation the nature of the inclusion $\operatorname{coh}\left(\mathcal{A}_{K}^{\lambda}\right) \subset \mathscr{C}_{\mathcal{A}_{K}^{\lambda}}$ measures, so to speak, the failure of the limit sheaf $\mathcal{A}_{K}^{\lambda}$ to be coherent. For more details we refer to the main body of this article. 
In the second part of this paper we apply the methods of the first part to prove an analogue of a classical theorem of S.P. Smith on complex universal enveloping algebras ([56]) in our locally analytic context. Let $L / \mathbb{Q}_{p}$ be an arbitrary finite extension and $G$ an arbitrary Lie group over $L$. The theory of admissible locally analytic $G$-representations provides a unified framework for studying finite-dimensional algebraic representations (if $G$ comes from an algebraic group) as well as the admissible-smooth representations of Langlands theory. Apart from the latter two classes there are many representations which are genuinely locally analytic. As a first coarse way to organize this situation and, more specifically, to meisure the 'size' of the vector space underlying a representation (which usually is of infinite vector space dimension) one may introduce an Auslander-Gorenstein style dimension function on the dual category $\mathscr{C}$ of coadmissible $D(G)$-modules (cf. [54]). It associates a well-defined number $0 \leq \operatorname{dim}(M) \leq d$ to each nonzero coadmissible module $M$ where $d$ equals the dimension of the Lie group $G$. In this way one obtains a filtration by Serre subcategories

$$
\mathscr{C}=\mathscr{C}_{d} \supseteq \mathscr{C}_{d-1} \supseteq \ldots \supseteq \mathscr{C}_{1} \supseteq \mathscr{C}_{0}
$$

where $M$ lies in $\mathscr{C}_{i}$ if and only if $\operatorname{dim}(M) \leq i$. The modules corresponding to the aforementioned algebraic representations and the smooth-admissible representations are concentrated in dimension zero.

Here, we investigate this situation under the additional assumption that the Lie algebra $\operatorname{Lie}(G)$ equals the Lie algebra of a split reductive group $\mathbf{G}$ satisfying our three hypotheses above. It turns out that, in this case, there is a large 'gap' in the above filtration. Namely, let $\mathbf{G}_{\mathbb{C}}^{\prime}$ be the complex derived group of $\mathbf{G}$ and let $r$ be half the smallest possible dimension of a non-zero co-adjoint $\mathbf{G}_{\mathbb{C}}^{\prime}$-orbit. The value of $r$ depends only on the root system of $\mathbf{G}_{\mathbb{C}}^{\prime}$ and is well-known in all cases according to work of A. Joseph ([28, [29]). Our main result says that if $M$ is a coadmissible $D(G)$-module which is not zero-dimensional, then $\operatorname{dim}(M) \geq r$. Moreover, we show that a coadmissible module is zero-dimensional if and only if its associated coherent sheaf (in the sense of [54]) consists of finite-dimensional $K$-vector spaces.

In [4] Ardakov-Wadsley prove a version of Smith's theorem for $p$-adically completed universal enveloping algebras and our version had its origin in the attempt to generalize their result to locally analytic representations. The results on locally analytic distribution algebras as obtained in [46] and [47] enable us to make a rather straightforward reduction to the case treated in [4].

The author thanks K. Ardakov for kindly answering some questions concerning the work [4] and for his comments on an earlier version of this article.

\footnotetext{
${ }^{1}$ In [loc.cit.] the authors consider the associated codimension function.
} 


\section{Preliminaries on CRossed Products AND SheAVES}

All appearing rings in this section are unital.

2.1. Recall ([43]) that a (associative) crossed product of a ring $R$ by a group $H$ is an associative ring $R * H$ which contains $R$ as a subring and contains a set of units $\bar{H}=\{\bar{h}: h \in H\}$, isomorphic as a set to $H$, such that

(a) $R * H$ is a free left $R$-module with basis $\bar{H}$;

(b) for all $x, y \in H, \bar{x} R=R \bar{x}$ and $\bar{x} \cdot \bar{y} R=\overline{x y} R$.

Given such a crossed product one obtains maps $\sigma: H \rightarrow \operatorname{Aut}(R)$ (an action) and $\tau$ : $H \times H \rightarrow R^{\times}$(a twisting) by the rules

$$
\sigma(x)(r)=\bar{x}^{-1} r \bar{x} \quad \text { and } \quad \bar{x} \cdot \bar{y}=\overline{x y} \tau(x, y) .
$$

It follows that $\sigma$ defines a group homomorphism $H \rightarrow \operatorname{Out}(R)$ and that $\tau$ is a 2-cocycle for the action of $H$ on $R^{\times}$via $\sigma 2$ Conversely, starting with a ring $R$, a group $H$, a group homomorphism $\sigma: H \rightarrow \operatorname{Out}(R)$ and a 2-cocycle $\tau: H \times H \rightarrow R^{\times}$one can construct an associative ring $R *_{\sigma, \tau} H$ which is a crossed product of $R$ by $H$ having the prescribed action and twisting ([43], Lemma 1.1).

Given a crossed product $S=R * H$ we can and will always assume that $\overline{1}$ equals the unit element in $S$ (loc.cit., $\S 1$ ) and that the inclusion $R \hookrightarrow S$ is unital. Moreover, if $R$ is left (right) noetherian and $H$ is finite, then $S$ is left (right) noetherian (loc.cit., Prop. 1.6).

Let from now on $K$ be a field of characteristic zero and $H$ a finite group. Let $S=R * H$ be a crossed product. We assume that $R, S$ are $K$-algebras and that $R \hookrightarrow S$ is a $K$-algebra homomorphism. The following lemma is due to K. Ardakov. I thank him for allowing me to reproduce it here.

Lemma 2.2. Let $R \rightarrow A$ be a ring homomorphism which is (left) flat and which factores through the inclusion $R \hookrightarrow S$. The resulting ring homomorphism $S \rightarrow A$ is (left) flat.

Proof. Let $M$ be a right $S$-module. It suffices to show $\operatorname{Tor}_{1}^{S}(M, A)=0$. Let $K[H]$ be the usual group algebra. There is a ring homomorphism $\Delta: S \rightarrow S \otimes_{K} K[H]$ induced by $\bar{h} \mapsto \bar{h} \otimes h$. The tensor product $M \otimes_{K} K[H]$ is a right $S$-module via $\Delta$. Now $\Delta$ exhibits $R \hookrightarrow S$ as a (right) $K[H]$-Galois extension (e.g. [3, 2.2). According to loc.cit., Prop. 2.3 (a) the $S$-module $M \otimes_{K} K[H]$ is therefore isomorphic to $M \otimes_{R} S$. On the other hand, the semisimplicity of $K[H]$ together with loc.cit., Lemma 2.4 implies that $M$ is an $S$-module direct summand of $M \otimes_{K} K[H]$. If $N$ is a complementary submodule we therefore obtain $\operatorname{Tor}_{1}^{S}(M, A) \oplus \operatorname{Tor}_{1}^{S}(N, A)=\operatorname{Tor}_{1}^{S}\left(M \otimes_{K} K[H], A\right) \simeq \operatorname{Tor}_{1}^{S}\left(M \otimes_{R} S, A\right)=\operatorname{Tor}_{1}^{R}(M, A)=0$ where the last two identities follow from flatness of $R \rightarrow S$ and $R \rightarrow A$ respectively.

\footnotetext{
${ }^{2}$ Note that we implicitly use the convention of loc.cit. for the multiplication in $\operatorname{Aut}(R)$ (and therefore also in $\operatorname{Out}(R)):(\alpha \beta)(r):=\beta(\alpha(r))$ for $r \in R$ and two automorphisms $\alpha, \beta$.
} 
2.3. Let $X$ be a topological space and $\mathcal{B}$ be a sheaf of not necessarily commutative rings on $X$. Let $H$ be a finite group. There is an obvious notion of (associative) crossed product of the sheaf $\mathcal{B}$ by $H$ by which we mean a sheaf $\mathcal{B} * H$ of associative rings on $X$ which contains $\mathcal{B}$ as a subsheaf and has a distinguished set of global units

$$
\bar{H}=\{\bar{h}: h \in H\} \subseteq \Gamma(X, \mathcal{B} * H)^{\times},
$$

isomorphic as a set to $H$, such that

(a) $\mathcal{B} * H$ is a free left $\mathcal{B}$-module with basis $\bar{H}$ (i.e. the natural map $\oplus_{|H|} \mathcal{B} \rightarrow \mathcal{B} * H$ given by the global sections $\bar{h}$ is an isomorphism of $\mathcal{B}$-modules),

(b) for all $x, y \in H, \bar{x} \mathcal{B}=\mathcal{B} \bar{x}$ and $\bar{x} \cdot \bar{y} \mathcal{B}=\overline{x y} \mathcal{B}$.

Given such a crossed product one obtains maps $\sigma: H \rightarrow \operatorname{Aut}(\mathcal{B})$ and $\tau: H \times H \rightarrow$ $\Gamma(X, \mathcal{B})^{\times}$as before, i.e. by the rules $\sigma(x)(s)=\bar{x}^{-1} s \bar{x}$ and $\bar{x} \cdot \bar{y}=\overline{x y} \tau(x, y)$ for any local section $s$. It follows that $\sigma$ defines a group homomorphism $H \rightarrow \operatorname{Out}(\mathcal{B})$ and $\tau$ is a 2 -cocycle for the action of $H$ on $\mathcal{B}^{\times}$via $\sigma$. Here, $\operatorname{Out}(\mathcal{B})$ refers to the quotient of $\operatorname{Aut}(\mathcal{B})$ by its normal subgroup of automorphisms arising via conjugation by a global unit. Furthermore, to match with our convention in the case of rings, we define here $(\alpha \beta)(s):=\beta(\alpha(s))$ for a local section $s$ of $\mathcal{B}$ and automorphisms $\alpha, \beta$.

Conversely, starting with a sheaf $\mathcal{B}$, a group $H$, a group homomorphism $\sigma: H \rightarrow \operatorname{Out}(\mathcal{B})$ and a 2-cocycle $\tau: H \times H \rightarrow \Gamma(X, \mathcal{B})^{\times}$one can construct a sheaf of associative rings $\mathcal{B} *_{\sigma, \tau} H$ which is a crossed product of $\mathcal{B}$ by $H$ having the prescribed action and twisting. The construction is a straightforward sheafification of the usual argument and is left to the reader.

Given a crossed product $\mathcal{B} * H$ we will always assume that $\overline{1}$ equals the global unit $1 \in \Gamma(X, \mathcal{B} * H)^{\times}$and that the inclusion $\mathcal{B} \hookrightarrow \mathcal{B} * H$ is unital.

2.4. Let again $\mathcal{B}$ be a sheaf of not necessarily commutative rings on $X$. Recall ([21], 0.5.3.1) that a left $\mathcal{B}$-module is coherent if it is of finite type and if for all open subsets $U \subset X$ and all morphisms $\varphi:\left.\left(\left.\mathcal{B}\right|_{U}\right)^{b} \rightarrow \mathcal{M}\right|_{U}$ the kernel of $\varphi$ is again of finite type. The coherent $\mathcal{B}$-modules form a full abelian subcategory $\operatorname{coh}(\mathcal{B})$ of all $\mathcal{B}$-modules. We say $\mathcal{B}$ is a sheaf of coherent rings if $\mathcal{B}$ is coherent as a left module over itself. If $\mathcal{B}$ is a sheaf of coherent rings, then a $\mathcal{B}$-module is coherent if and only if it is of finite presentation (loc.cit., 0.5.3.7).

2.5. We suppose we are now given a projective system of sheaves $\mathcal{B}_{n}, n \geq 0$ of not necessarily commutative $K$-algebras on $X$. We assume the following two local properties hold for the sheaves $\mathcal{B}_{n}$. Any point $x \in X$ has a basis $\mathcal{S}_{x}$ of open neighbourhoods $U \subseteq X$ such that

(i) $\Gamma\left(U, \mathcal{B}_{n}\right)$ is a left and right noetherian $K$-Banach algebra,

(ii) the transition homomorphism $\Gamma\left(U, \mathcal{B}_{n+1}\right) \rightarrow \Gamma\left(U, \mathcal{B}_{n}\right)$ is continuous and is left and right flat with dense image. 
We remark here that a straightforward generalization of [11], Prop. 1.2.1/2 to the noncommutative setting shows that the given norm in (i) can always be replaced by an equivalent one which is submultiplicative.

The union $\mathcal{S}:=\cup_{x \in X} \mathcal{S}_{x}$ is a base for the topology on $X$. We let

$$
\mathcal{B}:=\lim _{n} \mathcal{B}_{n}
$$

be the projective limit of the system $\left(\mathcal{B}_{n}\right)_{n}$. Note that $\Gamma(U, \mathcal{B})=\lim _{n} \Gamma\left(U, \mathcal{B}_{n}\right)$ for all open subsets $U \subseteq X([21], 0.3 .2 .6)$. In the following we often abbreviate $\mathcal{F}(U):=\Gamma(U, \mathcal{F})$ for a sheaf $\mathcal{F}$ on $X$ and an open subset $U \subseteq X$.

2.6. Let $\operatorname{Mod}(\mathcal{B})$ be the abelian category of (left) $\mathcal{B}$-modules on $X$. Our definitions allow the following simple sheafification of the formalism of coadmissible modules as developed in [54], §3. In particular, we will produce a certain full abelian subcategory

$$
\operatorname{coh}(\mathcal{B}) \subset \mathscr{C}_{\mathcal{B}} \subset \operatorname{Mod}(\mathcal{B})
$$

To do this consider the category of projective systems $\left(\mathcal{M}_{n}\right)_{n}$ of coherent $\mathcal{B}_{n}$-modules $\mathcal{M}_{n}$ with the property that the transition maps induce isomorphisms

$$
\mathcal{B}_{n} \otimes_{\mathcal{B}_{n+1}} \mathcal{M}_{n+1} \stackrel{\cong}{\longrightarrow} \mathcal{M}_{n}
$$

of $\mathcal{B}_{n}$-modules. With the usual notion of morphism these projective systems form a category $\operatorname{coh}\left(\left(\mathcal{B}_{n}\right)_{n}\right)$. As a consequence of the flatness requirement (ii) the base change functor

$$
\mathcal{B}_{n} \otimes_{\mathcal{B}_{n+1}}(\cdot): \operatorname{Mod}\left(\mathcal{B}_{n+1}\right) \rightarrow \operatorname{Mod}\left(\mathcal{B}_{n}\right)
$$

is exact. In view of the noetherian hypothesis in $(\mathrm{i})$ the category $\operatorname{coh}\left(\left(\mathcal{B}_{n}\right)_{n}\right)$ is therefore abelian. We have an additive and left exact functor

$$
\Gamma\left(\mathcal{M}_{n}\right):=\lim _{n} \mathcal{M}_{n}
$$

into $\operatorname{Mod}(\mathcal{B})$. Borrowing terminology from loc.cit. a $\mathcal{B}$-module $\mathcal{M}$ will be called coadmissible if it is isomorphic to a module of the form $\Gamma\left(\mathcal{M}_{n}\right)$ for some $\left(\mathcal{M}_{n}\right)_{n} \in \operatorname{coh}\left(\left(\mathcal{B}_{n}\right)_{n}\right)$. We let $\mathscr{C}_{\mathcal{B}}$ be the full subcategory of $\operatorname{Mod}(\mathcal{B})$ consisting of coadmissible modules.

Proposition 2.8. The functor $\Gamma$ is exact. For any $\left(\mathcal{M}_{n}\right)_{n}$ in $\operatorname{coh}\left(\left(\mathcal{B}_{n}\right)_{n}\right)$ and $M=\Gamma\left(\mathcal{M}_{n}\right)$ the natural map

$$
\mathcal{B}_{n} \otimes_{\mathcal{B}} \mathcal{M} \stackrel{\cong}{\longrightarrow} \mathcal{M}_{n}
$$

is an isomorphism for any $n \geq 0$. For any $U \in \mathcal{S}$ and for any $n \geq 0$ the ring homomorphism $\mathcal{B}(U) \rightarrow \mathcal{B}_{n}(U)$ is left and right flat.

Proof. This is a straightfoward adaption of the arguments in Theorem A and B and Cor. 3.1 of loc.cit. as follows. Given $\mathcal{M}_{n}$ and a point $x \in X$ there is an open neighbourhood $U$ of $x$ such that any $\mathcal{M}_{n}(U)$ is a finitely generated module over the noetherian Banach algebra $\mathcal{B}_{n}(U)$. It therefore has a canonical Banach topology (loc.cit., Prop. 2.1). By the density requirement in (ii) the projective system $\left(\mathcal{M}_{n}(U)\right)_{n}$ has the property that 
$\mathcal{M}_{m}(U) \rightarrow \mathcal{M}_{n}(U)$ has dense image for all $m>n$. In particular, the map $M(U) \rightarrow$ $\mathcal{M}_{n}(U)$ has dense image where $M:=\Gamma\left(\mathcal{M}_{n}\right)$. It now follows that, given a surjection $\left(\mathcal{M}_{n}\right)_{n} \rightarrow\left(\mathcal{N}_{n}\right)_{n}$ in $\operatorname{coh}\left(\left(\mathcal{B}_{n}\right)_{n}\right)$ the map $M(U) \rightarrow N(U)$ is surjective and

$$
\mathcal{B}_{n}(U) \otimes_{\mathcal{B}(U)} \mathcal{M}(U) \stackrel{\cong}{\longrightarrow} \mathcal{M}_{n}(U)
$$

is an isomorphism. Letting $U$ run through a neighbourhood basis for $x$ gives the first two claims. The last assertion follows similarly (cf. loc.cit., Remark 3.2).

Corollary 2.9. The functor

$$
\Gamma: \operatorname{coh}\left(\left(\mathcal{B}_{n}\right)_{n}\right) \stackrel{\cong}{\longrightarrow} \mathscr{C}_{\mathcal{B}}
$$

is an equivalence of categories.

Proof. By definition the functor $\Gamma: \operatorname{coh}\left(\left(\mathcal{B}_{n}\right)_{n}\right) \rightarrow \mathscr{C}_{\mathcal{B}}$ is essentially surjective. According to the preceding proposition it is fully faithful.

Proposition 2.10. Any $\mathcal{B}$-module of finite presentation is coadmissible. In particular, $\operatorname{coh}(\mathcal{B}) \subset \mathscr{C}_{\mathcal{B}}$

Proof. Let $V \subseteq X$ be an open set. By the local nature of our conditions imposed we may apply the above construction to the projective limit sheaf $\left.\mathcal{B}\right|_{V}=\lim _{\leftarrow_{n}}\left(\left.\mathcal{B}_{n}\right|_{V}\right)$ and obtain the abelian category of coadmissible $\left.\mathcal{B}\right|_{V}$-modules on $V$. Obviously, a given $\mathcal{B}$-module $\mathcal{M}$ is coadmissible if and only if this is true for $\left.\mathcal{M}\right|_{V}$ for all open sets $V \subseteq X$. Suppose $\mathcal{M}$ is of finite presentation. Locally, $\mathcal{M}$ equals the cokernel of a morphism of the type $\mathcal{B}^{a} \rightarrow \mathcal{B}^{b}$ for numbers $a, b \geq 0$ and is therefore coadmissible.

We finally suppose that our chosen base $\mathcal{S}$ for the topology on $X$ is such that

(iii) for all $U, V \in \mathcal{S}$ with $V \subset U$ the restriction homomorphism

$$
\Gamma\left(U, \mathcal{B}_{n}\right) \rightarrow \Gamma\left(V, \mathcal{B}_{n}\right)
$$

is left and right flat for all $n \geq 0$.

According to (i) and [7], Prop. (3.1.1) the sheaf $\mathcal{B}_{n}$ is then a sheaf of coherent rings for all $n$. Of course, this does not imply the coherence of the sheaf $\mathcal{B}$.

2.11. We keep the notation of the preceding paragraph. For each $n \geq 0$ we now assume additionally that we are given a finite group $H_{n}$ and a crossed product

$$
\mathcal{A}_{n}=\mathcal{B}_{n} *_{\sigma_{n}, \tau_{n}} H_{n}
$$

with the properties: $\mathcal{B}_{n} \hookrightarrow \mathcal{A}_{n}$ maps $K$ into the center and for $x \in X, U \in \mathcal{S}_{x}$ and $h \in H$ the algebra automorphism

$$
\sigma_{n}(h): \mathcal{B}_{n}(U) \stackrel{\cong}{\longrightarrow} \mathcal{B}_{n}(U)
$$

is an isometry of the Banach algebra $\mathcal{B}_{n}(U)$ with respect to a defining Banach norm on $\mathcal{B}_{n}(U)$ which is submultiplicative (cf. 2.5). We further suppose that this collection of sheaves $\left(\mathcal{A}_{n}\right)_{n}$ of $K$-algebras forms a projective system and that the transition morphism $\mathcal{A}_{n+1} \rightarrow \mathcal{A}_{n}$ is compatible with the map $\mathcal{B}_{n+1} \rightarrow \mathcal{B}_{n}$ for all $n$. 
Proposition 2.12. The system $\left(\mathcal{A}_{n}\right)_{n}$ satisfies conditions (i), (ii) and (iii).

Proof. Let $x \in X$ and $U \in \mathcal{S}_{x}$. We have $\mathcal{A}_{n}(U)=\mathcal{B}_{n}(U) * H_{n}$ which is a noetherian ring (cf. 2.1). Let |.| denote the chosen submultiplicative Banach norm on $\mathcal{B}_{n}(U)$. Then $\mathcal{A}_{n}(U)$ has a natural Banach space norm given by the maximum norm $q(\cdot)$ with respect to the $\mathcal{B}_{n}(U)$-module basis $\left\{\bar{h}: h \in H_{n}\right\}$. By assumption we have

$$
\left|\bar{h}^{-1} x \bar{h}\right|=\left|\sigma_{n}(h)(x)\right|=|x|
$$

for $h \in H_{n}, x \in \mathcal{B}_{n}(U)$. Let $\bar{h}_{1}, \ldots, \bar{h}_{s}$ be an enumeration of the finitely many elements $\left\{\bar{h}: h \in H_{n}\right\}$. Let us write $\mu=\sum_{k} \lambda_{k}(\mu) \bar{h}_{k}$ for an arbitrary element $\mu \in \mathcal{A}_{n}(U)$ with coefficients $\lambda_{k}(\mu) \in \mathcal{B}_{n}(U)$. In particular, $q(\mu)=\max _{k}\left|\lambda_{k}(\mu)\right|$. Applying this to the product $\bar{h}_{i} \bar{h}_{j} \in \mathcal{A}_{n}(U)$ defines coefficients $\lambda_{k}\left(\bar{h}_{i} \bar{h}_{j}\right)$. For two arbitrary elements $\mu, \mu^{\prime} \in \mathcal{A}_{n}(U)$ we now compute

$$
\begin{aligned}
\mu \mu^{\prime}=\sum_{i, j} \lambda_{i}(\mu) \bar{h}_{i} \lambda_{j}\left(\mu^{\prime}\right) \bar{h}_{j} & =\sum_{i, j} \lambda_{i}(\mu) \sigma_{n}\left(h_{i}\right)^{-1}\left(\lambda_{j}\left(\mu^{\prime}\right)\right) \bar{h}_{i} \bar{h}_{j} \\
& =\sum_{i, j, k} \lambda_{i}(\mu) \sigma_{n}\left(h_{i}\right)^{-1}\left(\lambda_{j}\left(\mu^{\prime}\right)\right) \lambda_{k}\left(\bar{h}_{i} \bar{h}_{j}\right) \bar{h}_{k} .
\end{aligned}
$$

For the value of $q\left(\mu \mu^{\prime}\right)$ we therefore find

$$
\begin{aligned}
\max _{i, j, k}\left|\lambda_{i}(\mu) \sigma_{n}\left(h_{i}\right)^{-1}\left(\lambda_{j}\left(\mu^{\prime}\right)\right) \lambda_{k}\left(\bar{h}_{i} \bar{h}_{j}\right)\right| & \leq \max _{i, j, k}\left|\lambda_{i}(\mu)\right| \cdot\left|\sigma_{n}\left(h_{i}\right)^{-1}\left(\lambda_{j}\left(\mu^{\prime}\right)\right)\right| \cdot\left|\lambda_{k}\left(\bar{h}_{i} \bar{h}_{j}\right)\right| \\
& \leq q(\mu) q\left(\mu^{\prime}\right) \max _{i, j, k}\left|\lambda_{k}\left(\bar{h}_{i} \bar{h}_{j}\right)\right|
\end{aligned}
$$

using (*) for the last inequality. This shows that the ring multiplication on $\mathcal{A}_{n}(U)$ is continuous with respect to the Banach topology coming from $q(\cdot)$. In other words, $\mathcal{A}_{n}(U)$ is a noetherian Banach algebra which yields (i). We furthermore have a commutative diagram of rings

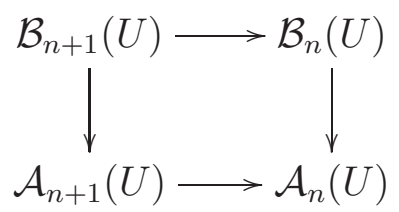

in which the upper horizontal arrow is flat with dense image and the vertical arrows are finite free ring extensions. By definition of the Banach topologies the lower horizontal arrow has dense image. According to Lemma 2.2 it is flat whence (ii). Applying loc.cit. to the lower horizontal arrow in the commutative diagram

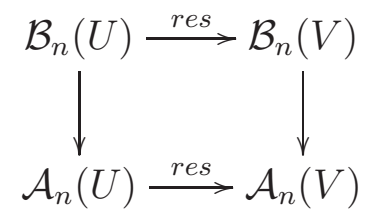

for $U, V \in \mathcal{S}$ with $V \subset U$ finally yields (iii). 
Let $\mathcal{A}:=\lim _{n} \mathcal{A}_{n}$. By the above proposition we therefore have an equivalence between abelian categories

$$
\Gamma: \operatorname{coh}\left(\left(\mathcal{A}_{n}\right)_{n}\right) \stackrel{\cong}{\longrightarrow} \mathscr{C}_{\mathcal{A}}
$$

and each sheaf $\mathcal{A}_{n}$ is coherent. Moreover, $\operatorname{coh}(\mathcal{A}) \subset \mathscr{C}_{\mathcal{A}}$.

\section{Differential operators on homogeneous spaCes}

From now on we will use the following notation: $p$ is a prime number in $\mathbb{Z}$ and $\mathbb{Q}_{p} \subseteq L \subseteq K$ denotes a chain of finite extensions of $\mathbb{Q}_{p}$. The absolute value $|$.$| on K$ is normalized by $|p|=p^{-1}$. Let $o_{L} \subseteq L$ be the ring of integers and $\pi_{L} \in o_{L}$ a uniformizer. Let $\kappa:=o_{L} /\left(\pi_{L}\right)$ denote the residue field of $L$. Let $\left[L: \mathbb{Q}_{p}\right]$ and $e$ be the degree and the ramification index of the extension $L / \mathbb{Q}_{p}$ respectively. Also, $o_{K} \subset K$ denotes the integers in $K$ and $\pi_{K} \in o_{K}$ a uniformizer. An $o_{K}$-submodule of a vector space $V$ over $K$ is called a lattice if it contains a basis of $V$ over $K$.

We recall some notions and constructions related to differential operators on homogeneous spaces $([4,[9],[10])$ thereby fixing some notation.

3.1. Let $\mathrm{G}$ be a connected split reductive group scheme over $o_{L}$. We denote its group of $o_{L}$-valued points by $\mathbf{G}\left(o_{L}\right)$. Let $\mathbf{T} \subset \mathbf{G}$ be a maximal torus with Lie algebra $\mathbf{t}$. Let $X^{*}(\mathbf{T})$ and $X_{*}(\mathbf{T})$ be the group of algebraic characters respectively cocharacters of $\mathbf{T}$ with the usual perfect pairing

$$
\langle\cdot, \cdot\rangle: X^{*}(\mathbf{T}) \times X_{*}(\mathbf{T}) \longrightarrow \mathbb{Z}
$$

defined by $z^{\langle a, b\rangle}:=a \circ b(z)$. Let $\Phi \subset X^{*}(\mathbf{T})$ denote the root system determined by the adjoint action of $\mathbf{T}$ on the Lie algebra $\mathfrak{g}$ of $\mathbf{G}$. Let $W$ denote the corresponding Weyl group. For any $w \in W$ we fix a representative $\dot{w}$ in $\mathbf{G}\left(o_{L}\right)$.

We choose a Borel subgroup scheme $\mathbf{B} \subset \mathbf{G}$ containing $\mathbf{T}$ and let $\Phi^{+} \subset \Phi$ be the associated subset of positive roots. We write $\mathbf{N}$ for the unipotent radical of $\mathbf{B}$. We identify, once and for all, the torus $\mathbf{T}$ with the abstract Cartan subgroup $\mathbf{B} / \mathbf{N}$ via the morphism $\mathbf{T} \subset \mathbf{B} \rightarrow \mathbf{B} / \mathbf{N}$ where the second map equals the canonical projection. The group $W$ acts naturally on the spaces $\mathfrak{t}^{*}:=\operatorname{Hom}_{o_{L}}\left(\mathfrak{t}, o_{L}\right), \mathfrak{t}_{L}^{*}:=\mathfrak{t}^{*} \otimes_{o_{L}} L$ and $\mathfrak{t}_{L}:=\mathfrak{t} \otimes_{o_{L}} L$. Via differentiation $d: X^{*}(\mathbf{T}) \hookrightarrow \mathfrak{t}^{*}$ we view $X^{*}(\mathbf{T})$ as a subgroup of $\mathfrak{t}^{*}$. We have that $X^{*}(\mathbf{T}) \otimes_{\mathbb{Z}} L=\mathfrak{t}_{L}^{*}$ and, via $\langle.,$.$\rangle and base change from \mathbb{Z}$ to $L$, that $X_{*}(\mathbf{T}) \otimes_{\mathbb{Z}} L=\mathfrak{t}_{L}$. Let $\rho=\frac{1}{2} \sum_{\alpha \in \Phi^{+}} \alpha$. Let $\check{\alpha}$ be the coroot of $\alpha \in \Phi$ viewed as an element of $\mathfrak{t}_{L}$. An arbitrary weight $\mu \in \mathfrak{t}_{L}^{*}$ is called dominant if

$$
\mu(\check{\alpha}) \notin\{-1,-2,-3, \ldots\}
$$

for all $\alpha \in \Phi^{+}$. The weight $\mu$ is called regular if its stabilizer under the $W$-action is trivial.

3.2. We write $\mathbf{N}$ for the unipotent radical of $\mathbf{B}$ and denote by $\mathbf{N}^{-}$the unipotent radical of the Borel subgroup scheme opposite to $\mathbf{B}$. The schemes $\mathbf{B}$ and $\mathbf{N}$ act via right translations on $\mathbf{G}$ and we put

$$
\tilde{X}:=\mathbf{G} / \mathbf{N}, \quad X:=\mathbf{G} / \mathbf{B}
$$


for the corresponding quotients. These are smooth and separated schemes over $o_{L}$. The canonical projection $\xi: \tilde{X} \longrightarrow X$ is a smooth morphism. Since $\mathbf{T}$ normalizes $\mathbf{N}$ the scheme $\tilde{X}$ has a right $\mathbf{T}$-action making $\xi$ a $\mathbf{T}$-torsor for the Zariski topology on $X$ (in the sense of [40], III. $\S 4$ ). Indeed, a covering of $X$ which trivializes $\xi$ is given by the open subschemes $\mathbf{U}_{w}, w \in W$ where

$$
\mathbf{U}_{w}:=\text { image of } \dot{w} \mathbf{N}^{-} \mathbf{B}
$$

under the canonical projection $\mathbf{G} \rightarrow \mathbf{G} / \mathbf{B}([27$, II.(1.10)). We let $\mathcal{S}$ denote the collection of affine open subsets of $X$ over which $\xi$ becomes trivial. By what we have just said it is a base for the Zariski topology on $X$.

3.3. Let $\mathcal{O}_{\tilde{X}}$ be the structure sheaf of $\tilde{X}$ and let $\mathcal{T}_{\tilde{X}}=\mathcal{D} e r_{o_{L}}\left(\mathcal{O}_{\tilde{X}}\right)$ be the tangent sheaf of $\tilde{X}([23], 16.5 .7)$. Using the natural action of $\mathcal{T}_{\tilde{X}}$ on $\mathcal{O}_{\tilde{X}}$ by derivations we have the semidirect product $\mathcal{O}_{\tilde{X}} \oplus \mathcal{T}_{\tilde{X}}$ of $\mathcal{T}_{\tilde{X}}$ with the abelian Lie algebra $\mathcal{O}_{\tilde{X}}$, a sheaf of Lie algebras over $o_{L}$. The corresponding universal enveloping algebra is called the sheaf of crystalline differential operators (of level zero) on $\tilde{X}\left([8]\right.$, [9]). Following [4], 4.2 we denote it by $\mathcal{D}_{\tilde{X}}$. It is a sheaf of $o_{L}$-algebras and, at the same time, an $\mathcal{O}_{\tilde{X}}$-module through the map

$$
\mathcal{O}_{\tilde{X}} \longrightarrow \mathcal{O}_{\tilde{X}} \oplus \mathcal{T}_{\tilde{X}}, f \mapsto(f, 0) \text {. }
$$

There is a positive increasing $\mathbb{Z}$-filtration on $\mathcal{D}_{\tilde{X}}$

$$
0 \subset F_{0} \mathcal{D}_{\tilde{X}} \subset F_{1} \mathcal{D}_{\tilde{X}} \subset F_{2} \mathcal{D}_{\tilde{X}} \subset \cdots
$$

consisting of coherent $\mathcal{O}_{\tilde{X}^{-} \text {-submodules, such that }}$

$$
F_{0} \mathcal{D}_{\tilde{X}}=\mathcal{O}_{\tilde{X}}, \quad F_{1} \mathcal{D}_{\tilde{X}}=\mathcal{O}_{\tilde{X}} \oplus \mathcal{T}_{\tilde{X}} \quad \text { and } \quad F_{m} \mathcal{D}_{\tilde{X}}=F_{1} \mathcal{D}_{\tilde{X}} \cdot F_{m-1} \mathcal{D}_{\tilde{X}} \quad \text { for } m>1 .
$$

Given two local sections $\partial$ and $f$ of $\mathcal{T}_{\tilde{X}}$ and $\mathcal{O}_{\tilde{X}}$ respectively we have

$$
\partial \cdot f-f \cdot \partial=\partial(f)
$$

for their commutator in $\mathcal{D}_{\tilde{X}}$. This means that the associated graded sheaf of $\mathcal{D}_{\tilde{X}}$ is canonically isomorphic to the symmetric algebra of the locally free $\mathcal{O}_{\tilde{X}}$-module $\mathcal{T}_{\tilde{X}}$,

$$
g r \cdot \mathcal{D}_{\tilde{X}} \stackrel{\cong}{\longrightarrow} \operatorname{Sym}_{\mathcal{O}_{\tilde{X}}} \mathcal{T}_{\tilde{X}}
$$

We have an obvious morphism of $\mathcal{D}_{\tilde{X}}$ to the sheaf of usual differential operators on $\tilde{X}$ (23], 16.8). Since $\operatorname{char}(L)=0$ it is an isomorphism over the generic fibre of $\tilde{X}$.

By the same token there are corresponding sheaves of crystalline differential operators $\mathcal{D}_{X}$ and $\mathcal{D}_{\mathbf{T}}$ on the smooth $o_{L}$-schemes $X$ and $\mathbf{T}$ respectively.

3.5. We let

$$
\tilde{\mathcal{D}}:=\left(\xi_{*}\left(\mathcal{D}_{\tilde{X}}\right)\right)^{\mathbf{T}}
$$

denote the Borho-Brylinski relative enveloping algebra of the T-torsor $\xi([10], \S 1)$. Again, this is a sheaf of $o_{L}$-algebras and, at the same time, an $\mathcal{O}_{X}$-module through the map

$$
\xi^{\sharp}: \mathcal{O}_{X} \longrightarrow\left(\xi_{*} \mathcal{O}_{\tilde{X}}\right)^{\mathbf{T}} \text {. }
$$


It has a positive increasing $\mathbb{Z}$-filtration

$$
F_{m} \tilde{\mathcal{D}}:=\left(\xi_{*}\left(F_{m} \mathcal{D}_{\tilde{X}}\right)\right)^{\mathbf{T}}
$$

induced by the filtration $F_{\bullet} \mathcal{D}_{\tilde{X}}$. Given an open subset $U$ from $\mathcal{S}$ a choice of trivialization

$$
\xi^{-1}(U) \simeq U \times_{o_{L}} \mathbf{T}
$$

over $U$ induces an isomorphism of $o_{L^{-}}$algebras

$$
\tilde{\mathcal{D}}(U) \stackrel{\cong}{\longrightarrow} \mathcal{D}\left(U \times_{o_{L}} \mathbf{T}\right)^{\mathbf{T}}=\mathcal{D}_{X}(U) \otimes_{o_{L}} U(\mathfrak{t})
$$

where the right-hand side is the usual tensor product of $o_{L}$-algebras and where we use the symbol $\mathcal{D}$ to denote the crystalline differential operators on the smooth $o_{L}$-scheme $U \times_{o_{L}} \mathbf{T}$. The last identity follows here from

$$
\mathcal{D}\left(U \times_{o_{L}} \mathbf{T}\right)^{\mathbf{T}}=\left(\mathcal{D}_{X}(U) \otimes_{o_{L}} \mathcal{D}_{\mathbf{T}}(\mathbf{T})\right)^{\mathbf{T}}=\mathcal{D}_{X}(U) \otimes_{o_{L}} \mathcal{D}_{\mathbf{T}}(\mathbf{T})^{\mathbf{T}}
$$

using ([27], I.2.10(3)) together with the well-known isomorphism $U(\mathfrak{t}) \stackrel{\cong}{\longrightarrow} \mathcal{D}_{\mathbf{T}}(\mathbf{T})^{\mathbf{T}}$ for the split torus $\mathbf{T}$ (loc.cit., I.7.13).

3.6. The group $\mathbf{G}$ acts on $\tilde{X}$ and $X$ by left translations and $\xi$ is $\mathbf{G}$-equivariant. The sheaf $\mathcal{D}_{\tilde{X}}$ has a natural G-equivariant structure inherited from the usual equivariant structures of $\mathcal{O}_{\tilde{X}}$ and $\mathcal{T}_{\tilde{X}}$. Since the right $\mathbf{T}$-action on $\tilde{X}$ commutes with the left $\mathbf{G}$-action the sheaf $\tilde{\mathcal{D}}$ is naturally $\mathbf{G}$-equivariant.

In the following we denote the constant sheaf with fiber $U(\mathfrak{g})$ on $\tilde{X}$ again by $U(\mathfrak{g}$ ) (and similarly in other cases). Differentiating the left $\mathbf{G}$-action on $\tilde{X}$ gives a homomorphism $U(\mathfrak{g}) \rightarrow \mathcal{D}_{\tilde{X}}$ which is $\mathbf{G}$-equivariant with respect to the adjoint action of $\mathbf{G}$ on $U(\mathfrak{g})$ ([16], II.§4.4.4). Whenever the open set $V \subset \tilde{X}$ is right $\mathbf{T}$-stable the section of this morphism over $V$ has image in $\mathcal{D}_{\tilde{X}}(V)^{\mathbf{T}}$. Letting $V$ run through the subsets $\xi^{-1}(U)$ for $U \in \mathcal{S}$ one obtains a morphism

$$
\varphi: U(\mathfrak{g}) \longrightarrow \tilde{\mathcal{D}}
$$

which is $\mathbf{G}$-equivariant with respect to the adjoint action of $\mathbf{G}$ on $U(\mathfrak{g})$. Similarly, differentiating the right $\mathbf{T}$-action on $\tilde{X}$ induces a homomorphism

$$
\psi: U(\mathfrak{t}) \longrightarrow \tilde{\mathcal{D}}
$$

which is a central embedding. Its image lies in the G-invariants of $\tilde{\mathcal{D}}([38], \S 3)$.

\section{Deformations And COMpletions}

We keep the notation from the preceding section and recall a Beilinson-Bernstein type equivalence of categories for $p$-adically completed universal enveloping algebras as constructed in [4. 
4.1. Let as above $\mathfrak{g}$ and $\mathfrak{t}$ be the $o_{L}$-Lie algebras of the group schemes $\mathbf{G}$ and $\mathbf{T}$ respectively. Fix a number $n \geq 0$. We denote by $U(\cdot)$ the universal enveloping algebra of whatsoever Lie algebra we wish to consider. Choosing an $o_{L}$-basis for $\mathfrak{g}$ the algebra $U(\mathfrak{g})$ is endowed with a positive filtration $F_{\bullet} U(\mathfrak{g})$, its usual PBW-filtration ([17], 2.3). The associated graded algebra equals the symmetric algebra $S(\mathfrak{g})$ on $\mathfrak{g}$. According to these properties $U(\mathfrak{g})$ is therefore a deformable $o_{L^{-}}$-algebra in the sense of [4], Def. 3.5. Its $n$-th deformation (loc.cit.) is the $o_{L}$-submodule

$$
U(\mathfrak{g})_{n}:=\sum_{i \geq 0} \pi_{L}^{i n} F_{i} U(\mathfrak{g})
$$

of $U(\mathfrak{g})$. It is easily seen to be equal to $U\left(\pi_{L}^{n} \mathfrak{g}\right)$ and is therefore even a subalgebra of $U(\mathfrak{g})$. We denote by

$$
\widehat{U(\mathfrak{g})_{n}}={\underset{\lim }{m}} U(\mathfrak{g})_{n} / p^{m} U(\mathfrak{g})_{n}
$$

its $p$-adic completion. In the same way we have the algebra $U(\mathfrak{t})_{n}$ and its $p$-adic completion $\widehat{U(\mathfrak{t})_{n}}$.

4.2. As already recalled above the group $\mathbf{G}$ acts on $U(\mathfrak{g})$ by the usual adjoint representation. The ring of invariants $U(\mathfrak{g})^{\mathbf{G}}$ has the induced PBW-filtration from $U(\mathfrak{g})$ and we may form

$$
\left(U(\mathfrak{g})^{\mathbf{G}}\right)_{n}:=\sum_{i \geq 0} \pi_{L}^{i n} F_{i} U(\mathfrak{g})^{\mathbf{G}} .
$$

It is a subalgebra of $U(\mathfrak{g})_{n}$. Let $\mathfrak{n}$ and $\mathfrak{n}^{-}$be the Lie algebras of $\mathbf{N}$ and $\mathbf{N}^{-}$respectively. The triangular decomposition $\mathfrak{g}=\mathfrak{n}^{-} \oplus \mathfrak{t} \oplus \mathfrak{n}$ induces the linear projection $U(\mathfrak{g}) \rightarrow U(\mathfrak{t})$ with kernel $\mathfrak{n}^{-} U(\mathfrak{g})+U(\mathfrak{g}) \mathfrak{n}$. As in characteristic zero one shows that its restriction to the zero weight space with respect to the adjoint action of $\mathbf{T}$ on $U(\mathfrak{g})$ is an algebra homomorphism. We therefore have an algebra homomorphism

$$
\phi: U(\mathfrak{g})^{\mathbf{G}} \rightarrow U(\mathfrak{t})
$$

(cf. also [26], $§ 9$ for the corresponding construction over a field of positive characteristic). It extends to a homomorphism

$$
\phi_{n}:\left(U(\mathfrak{g})^{\mathbf{G}}\right)_{n} \longrightarrow U(\mathfrak{t})_{n} .
$$

For all that follows we fix a linear homomorphism

$$
\lambda \in \operatorname{Hom}_{o_{L}}\left(\pi_{L}^{n} \mathfrak{t}, o_{K}\right)
$$

and view it as a character $U(\mathfrak{t})_{n} \rightarrow o_{K}$. Using $\phi_{n}$ we may form the algebra

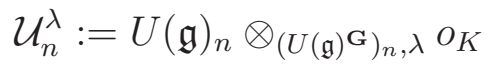

and its $p$-adic completion $\widehat{\mathcal{U}_{n}^{\lambda}}$. We let $\widehat{\mathcal{U}_{n}^{\lambda}},=\widehat{\mathcal{U}}_{n}^{\lambda} \otimes_{o_{K}} K$. Since $U\left(\pi_{L}^{n} \mathfrak{g}\right)$ is left and right noetherian, the rings $\widehat{\mathcal{U}_{n}^{\lambda}}$ and $\widehat{\mathcal{U}_{n}^{\lambda}}, K$ are left and right noetherian ([7], (3.2.2) (iii)). 
Remark: In [4] the authors consider only the case $\lambda\left(\pi_{L}^{n} \mathfrak{t}\right) \subseteq o_{L}$. However, all constructions of loc.cit. immediately generalize to the slightly more general case considered here.

4.4. Recall the relative universal enveloping algebra $\tilde{\mathcal{D}}$ of the torsor $\xi$. Let $U \in \mathcal{S}$ and choose a trivialization $\xi^{-1}(U) \simeq U \times_{o_{L}} \mathbf{T}$. The induced isomorphism

$$
\tilde{\mathcal{D}}(U) \simeq \mathcal{D}_{X}(U) \otimes_{o_{L}} U(\mathfrak{t})
$$

translates the positive $\mathbb{Z}$-filtration on the source into the tensor product filtration on the target (where the second factor in the target has its usual PBW-filtration). Thus, $\tilde{\mathcal{D}}(U)$ is a deformable algebra and hence, there is a $n$-th deformation

$$
\tilde{\mathcal{D}}(U)_{n}:=\sum_{i \geq 0} \pi_{L}^{i n} F_{i} \tilde{\mathcal{D}}(U) .
$$

We remark in passing that (4.5) induces an algebra isomorphism

$$
\tilde{\mathcal{D}}(U)_{n} \simeq \mathcal{D}_{X}(U)_{n} \otimes_{o_{L}} U(\mathfrak{t})_{n} .
$$

The formation $U \mapsto \tilde{\mathcal{D}}(U)_{n}$ yields a presheaf on the basis $\mathcal{S}$ of $X$. The sheafification functor produces therefore a sheaf $\tilde{\mathcal{D}}_{n}$ on $X$ whose algebra of sections over any $U \in \mathcal{S}$ coincides with $\tilde{\mathcal{D}}(U)_{n}$ (e.g. [11], last remarks in 9.2.1). Similarly, there is a sheaf $\widehat{\tilde{\mathcal{D}}}_{n}$ on $X$ whose algebra of sections over any $U \in \mathcal{S}$ equals the $p$-adic completion of $\tilde{\mathcal{D}}(U)_{n}$, i.e.

$$
\widehat{\tilde{\mathcal{D}}}_{n}(U) \simeq \widehat{\mathcal{D}_{X}(U)_{n}} \hat{\otimes}_{o_{L}} \widehat{U(\mathfrak{t})_{n}} .
$$

The formation of the sheaf $\widehat{\tilde{\mathcal{D}}}_{n}$ is compatible with the homomorphisms $\varphi$ and $\psi$ (cf. (3.7) and (3.8)) whence two homomorphisms

$$
\widehat{\varphi_{n}}: \widehat{U(\mathfrak{g})_{n}} \longrightarrow \widehat{\tilde{\mathcal{D}}_{n}} \quad \text { and } \quad \widehat{\psi_{n}}: \widehat{U(\mathfrak{t})_{n}} \longrightarrow \widehat{\tilde{\mathcal{D}}}_{n}
$$

The second map remains a central embedding. We therefore may form the central reduction

$$
\widehat{\mathcal{D}_{n}^{\lambda}}:=\widehat{\widetilde{\mathcal{D}}}_{n} \otimes_{\widehat{U(t) n}, \lambda} o_{K} .
$$

It is a major technical result in [4] (loc.cit, Lemma 4.10) that these two maps are related via the $p$-adic completion of the deformed 'Harish-Chandra homorphism' (4.3)

$$
\widehat{\phi_{n}}:\left(\widehat{U(\mathfrak{g})^{\mathbf{G}}}\right)_{n} \longrightarrow \widehat{U(\mathfrak{t})_{n}} .
$$

This yields a morphism of sheaves of algebras

$$
\widehat{\varphi_{n}^{\lambda}}: \widehat{\mathcal{U}_{n}^{\lambda}}=\widehat{U(\mathfrak{g})_{n}} \otimes_{\left(U(\mathfrak{g})^{\mathbf{G}}\right)_{n}, \lambda} o_{K} \longrightarrow \widehat{\tilde{\mathcal{D}}_{n}} \otimes_{\widehat{U(\mathfrak{t})_{n}, \lambda}} o_{K}=\widehat{\mathcal{\mathcal { D }}_{n}^{\lambda}} .
$$

We denote the base change to $K$ of these sheaves by $\widehat{\mathcal{D}_{n, K}^{\lambda}}$ and $\widehat{\mathcal{U}_{n}^{\lambda}, K}$ respectively and obtain a morphism $\widehat{\varphi_{n, K}^{\lambda}}: \widehat{\mathcal{U}_{n, K}^{\lambda}} \rightarrow \widehat{\mathcal{D}}_{n, K}^{\lambda}$. In the following we will regard $\widehat{\mathcal{D}_{n, K}^{\lambda}}$ as a module 
over $\widehat{\mathcal{U}_{n, K}^{\lambda}}$ via this map. Note that $\widehat{\mathcal{D}_{n, K}^{\lambda}}$ is a sheaf of coherent rings (loc.cit., Prop. 6.5 (d)).

4.8. We have the abelian categories $\operatorname{coh}\left(\widehat{\mathcal{D}}_{n, K}^{\lambda}\right)$ and $\operatorname{coh}\left(\Gamma\left(\widehat{\mathcal{D}_{n, K}^{\lambda}}\right)\right)$ of coherent (left) $\widehat{\mathcal{D}}_{n, K^{-}}^{-}$ modules respectively $\Gamma\left(\widehat{\mathcal{D}_{n, K}^{\lambda}}\right)$-modules. Given a $\Gamma\left(\widehat{\mathcal{D}_{n, K}^{\lambda}}\right)$-module $M$ we may form the $\widehat{\mathcal{D}_{n, K}^{\lambda}}$-module

$$
\operatorname{Loc}_{\lambda}(M):=\widehat{\mathcal{D}}_{n, K}^{\lambda} \otimes_{\Gamma\left(\widehat{\mathcal{D}}_{n, K}^{\lambda}\right)} M .
$$

Conversely, the module of global sections $\Gamma(\mathcal{M})$ of a $\widehat{\mathcal{D}_{n, K^{\lambda}}}$-module $\mathcal{M}$ is of course a $\Gamma\left(\widehat{\mathcal{D}_{n, K}^{\lambda}}\right)$-module. The functors $\left(\operatorname{Loc}_{\lambda}, \Gamma(\cdot)\right)$ form an adjoint pair. The following result may be viewed as a $p$-adically completed version of the théorème principal of [5], restricted to coherent modules. It is one of the main results of [4]. The case $n=0$ was already obtained in an earlier paper by Noot-Huyghe ([4]]). Recall that we have fixed a homomorphism $\lambda \in \operatorname{Hom}_{o_{L}}\left(\pi_{L}^{n} \mathfrak{t}, o_{K}\right)$.

Theorem 4.9. (Ardakov-Wadsley, Noot-Huyghe) Suppose the weight $\lambda+\rho \in \mathfrak{t}_{K}^{*}$ is dominant and regular. The adjoint pair $\left(\operatorname{Loc}_{\lambda}, \Gamma(\cdot)\right)$ induces mutually inverse equivalences of categories

$$
\operatorname{coh}\left(\Gamma\left(\widehat{\mathcal{D}_{n, K}^{\lambda}}\right)\right) \stackrel{\cong}{\longrightarrow} \operatorname{coh}\left(\widehat{\mathcal{D}}_{n, K}^{\lambda}\right) .
$$

4.10. Let $\bar{\kappa}$ be an algebraic closure of $\kappa$. Let us consider the following three hypothesis on the geometric closed fibre $\mathbf{G}_{\bar{s}}=\mathbf{G} \otimes_{o_{L}} \bar{\kappa}$ of $\mathbf{G}$ which are familiar from the theory of modular Lie algebras (cf. [26], 6.3).

(H1) The derived group of $\mathbf{G}_{\bar{s}}$ is (semisimple) simply connected.

(H2) The prime $p$ is good for the $\bar{\kappa}$-Lie algebra $\operatorname{Lie}\left(\mathbf{G}_{\bar{s}}\right)$.

(H3) There exists a $\mathbf{G}_{\bar{s}}$-invariant non-degenerate bilinear form on $\operatorname{Lie}\left(\mathbf{G}_{\bar{s}}\right)$.

A prominent example satisfying these conditions for all primes $p$ is the general linear group (using the trace form for (H3)). Any almost simple and simply connected $\mathbf{G}_{\bar{s}}$ satisfies these conditions if $p \geq 7$ (and if $p$ does not divide $n+1$ in case $\mathbf{G}_{\bar{s}}$ is of type $A_{n}$ ). For a more detailed discussion of these conditions we refer to loc.cit.

The next theorem is a version of [4], Thm. 6.10. It is proved in loc.cit. under the assumptions that $\mathbf{G}$ is semisimple and simply connected and that the prime $p$ is very good (in the sense of loc.cit., 6.8).

Theorem 4.11. (Ardakov-Wadsley) Assuming (H1)-(H3) the map $\widehat{\varphi_{n, K}^{\lambda}}$ induces an algebra isomorphism

$$
\widehat{\varphi_{n, K}^{\lambda}}: \widehat{\mathcal{U}_{n, K}^{\lambda}} \stackrel{\cong}{\longrightarrow} \Gamma\left(X, \widehat{\mathcal{D}_{n, K}^{\lambda}}\right) .
$$

Proof. Let $\mathfrak{t}_{\mathbb{Z}}$ denote the Lie algebra of the canonical extension of the torus $\mathbf{T}$ to a group scheme over $\mathbb{Z}$, and let $\rho$ be the map $\Phi^{\vee} \stackrel{\cong}{\longrightarrow} \Phi \subset \mathfrak{t}_{\mathbb{Z}}^{\vee}$ between coroots, roots and the dual of the free $\mathbb{Z}$-module $\mathfrak{t}_{\mathbb{Z}}$ which comes from the root datum of $\mathbf{G}$. The triple $\mathscr{R}=\left(\mathfrak{t}_{\mathbb{Z}}, \Phi^{\vee}, \rho\right)$ 
is then a système de racines (réduit) précisé in the sense of [15]. The assumptions in [4] are made in order to be able to apply [15], §6. Corollaire du Théorème 2 and Théorème 3 to $\mathscr{R}$ and the commutative ring $\mathbb{F}_{p}$ as well as to be able to apply [9], Prop. 3.4.1. However, these results are also available under the hypotheses $(\mathrm{H} 1)-(\mathrm{H} 3)$. Indeed, let $t_{\mathscr{R}}$ be the torsion index of $\mathscr{R}([15], \S 5)$ and assume (H1)-(H3). The discussion in [26], 9.6 shows that $t_{\mathscr{R}}$ is invertible in $\mathbb{F}_{p}$ and that the existence of an $\alpha^{\vee} \in \Phi^{\vee}$ with $\alpha^{\vee} / 2 \in \mathfrak{t}_{\mathbb{Z}}$ implies $p \neq 2$. Hence, [15], Corollaire du Théorème 2 and Théorème 3 apply to $\mathscr{R}$ and the commutative ring $\mathbb{F}_{p}$. Furthermore, the required statement from [9] extends to the case of $\mathbf{G}$ since it depends only on the derived group of $\mathbf{G}_{\bar{s}}$. Using these inputs the argumentation in [4], 6.9/10 goes through.

Remark: Under the hypotheses (H1)-(H3) and $\lambda+\rho$ being dominant and regular, the isomorphism (4.12) together with Thm. 4.9) implies an equivalence of categories $\operatorname{coh}\left(\widehat{\mathcal{D}_{n, K}^{\lambda}}\right) \simeq$ $\operatorname{Mod}^{\mathrm{fg}}\left(\widehat{\mathcal{U}_{n, K}^{\lambda}}\right)$ where the right-hand side denotes the finitely generated (left) modules over the noetherian $\operatorname{ring} \widehat{\mathcal{U}_{n, K}^{\lambda}}$.

4.13. We now study how the family of sheaves $\widehat{\mathcal{D}}_{n, K}^{\lambda}$ on $X$ varies in the deformation parameter $n \geq 0$. Let $U \in \mathcal{S}$. The inclusions

$$
F_{i} \tilde{\mathcal{D}}_{n+1}(U)=\pi_{L}^{i(n+1)} F_{i} \tilde{\mathcal{D}}(U) \subseteq \pi_{L}^{i n} F_{i} \tilde{\mathcal{D}}(U)=F_{i} \tilde{\mathcal{D}}_{n}(U)
$$

for all $i \geq 0$ induce a morphism of sheaves of algebras $\tilde{\mathcal{D}}_{n+1} \rightarrow \tilde{\mathcal{D}}_{n}$ for all $n \geq 0$. It extends to a morphism

$$
r e s_{\mathcal{D}}: \widehat{\mathcal{D}_{n+1, K}^{\lambda}} \longrightarrow \widehat{\mathcal{D}_{n, K}^{\lambda}}
$$

and yields a projective system $\left(\widehat{\mathcal{D}_{n, K}^{\lambda}}\right)_{n}, n \geq 0$.

Proposition 4.15. Let $U, V \in \mathcal{S}$ with $V \subset U$ and $n \geq 0$.

(i) Each $\Gamma\left(U, \widehat{\mathcal{D}_{n, K}^{\lambda}}\right)$ is a left and right noetherian Banach algebra,

(ii) the transition homomorphism $\Gamma\left(U, \widehat{\mathcal{D}_{n+1, K}^{\lambda}}\right) \rightarrow \Gamma\left(U, \widehat{\mathcal{D}_{n, K}^{\lambda}}\right)$ is left and right flat with dense image,

(iii) the restriction homomorphism $\Gamma\left(U, \widehat{\mathcal{D}_{n, K}^{\lambda}}\right) \rightarrow \Gamma\left(V, \widehat{\mathcal{D}_{n, K}^{\lambda}}\right)$ is left and right flat.

Proof. Being a $p$-adic completion the ring $\Gamma\left(U, \widehat{\mathcal{D}_{n}^{\lambda}}\right)$ is $p$-adically complete and separated. According to [7], (3.2.3) (iv),(vi) it is flat over $o_{K}$ and left and right noetherian. In particuar, it is a lattice in the $K$-vector space $\Gamma\left(U, \widehat{\mathcal{D}_{n, K}^{\lambda}}\right)=\Gamma\left(U, \widehat{\mathcal{D}_{n}^{\lambda}}\right) \otimes_{o_{K}} K$ and the corresponding gauge norm $([49]$, 2 ) makes the latter a (left and right noetherian) $K$ Banach algebra. This shows (i). The flatness property (iii) follows from the proof of [4], Prop. 5.9 (c) together with the last part of loc.cit., Prop. 6.5 (a). Let us establish property (ii). Since $X$ is smooth we may assume, by passing to a smaller $U \in \mathcal{S}$, that the tangent sheaf $\left.\mathcal{T}_{X}\right|_{U}$ is a free $\left.\mathcal{O}_{X}\right|_{U}$-module. We choose local coordinates $t_{1}, \ldots, t_{r}$ on $U$ 
and let $\partial_{1}, \ldots, \partial_{r}$ be the corresponding derivations. According to (3.4) we have an algebra isomorphism

$$
g r \cdot \mathcal{D}_{X}(U) \stackrel{\cong}{\longrightarrow} \mathcal{O}_{X}(U)\left[T_{1} \ldots, T_{r}\right], \quad \text { principal symbol of } \partial_{i} \mapsto T_{i}
$$

onto the polynomial ring in the variables $T_{i}$ with coefficients in $\mathcal{O}_{X}(U)$. Now consider the sheaf

$$
\mathcal{D}_{n}^{\lambda}:=\tilde{\mathcal{D}}_{n} \otimes_{U(\mathfrak{t})_{n}, \lambda} O_{K}
$$

on $X$. It follows from [4, Lemma 6.5 that the algebra of sections $\widehat{\mathcal{D}_{n}^{\lambda}}(U)$ over an open set $U \in \mathcal{S}$ equals the $p$-adic completion of $\mathcal{D}_{n}^{\lambda}(U)$. By general results on adic completions of non-commutative rings (e.g [7], (3.2.3) (vii)) we are therefore reduced to prove that the homomorphism $\mathcal{D}_{n+1}^{\lambda}(U) \rightarrow \mathcal{D}_{n}^{\lambda}(U)$ is flat. To do this choose a trivialization

$$
\xi^{-1}(U) \simeq U \times_{o_{L}} \mathbf{T}
$$

of the torsor $\xi$ over $U$. The isomorphism (4.7) is compatible with variation in $n$. Applying the toral character $\lambda$ we are reduced to prove that

$$
\mathcal{D}_{X}(U)_{n+1} \subseteq \mathcal{D}_{X}(U)_{n}
$$

is a flat homomorphism. Now $\mathcal{D}_{X}(U)_{m} \subseteq \mathcal{D}_{X}(U)$ has the subspace filtration for all $m \geq 0$ and the inclusion $(*)$ is therefore a filtered morphism. The induced $P B W$-filtration on source and target is positive, hence complete. By general principles (e.g. [54], Prop. 1.2) we are therefore reduced to prove flatness of the graded map

$$
g r \cdot \mathcal{D}_{n+1}(U) \subseteq g r \cdot \mathcal{D}_{n}(U) .
$$

By choice of $t_{1}, . ., t_{r}$ this map equals the natural inclusion

$$
\mathcal{O}_{X}(U)\left[\pi_{L}^{n+1} T_{1}, \ldots, \pi_{L}^{n+1} T_{r}\right] \subseteq \mathcal{O}_{X}(U)\left[\pi_{L}^{n} T_{1}, \ldots, \pi_{L}^{n} T_{r}\right] .
$$

Since source and target are flat over the discrete valuation ring $o_{L}$ the assertion follows by a straightforward application of the fiber criterion for flatness ([22], 11.3.10). To prove the statement about the image we consider the sheaf

$$
\mathcal{D}_{K}^{\lambda}:=\mathcal{D}_{0}^{\lambda} \otimes_{o_{K}} K=\tilde{\mathcal{D}} \otimes_{U\left(\mathfrak{t}_{K}\right), \lambda_{K}} K .
$$

It is obvious from (4.6) that $\tilde{\mathcal{D}} \otimes_{o_{K}} K=\tilde{\mathcal{D}}_{n} \otimes_{o_{K}} K$ and thus $\mathcal{D}_{K}^{\lambda}=\mathcal{D}_{n}^{\lambda} \otimes_{o_{K}} K$ holds for all $n \geq 0$. We deduce that the canonical homomorphism

$$
\mathcal{D}_{K}^{\lambda}(U) \longrightarrow \widehat{\mathcal{D}}_{n, K}^{\lambda}(U)
$$

has dense image for all $n$ and is compatible with variation in $n$. This proves the assertion.

Corollary 4.16. The projective system of sheaves $\left(\widehat{\mathcal{D}_{n, K}^{\lambda}}\right)_{n \geq 0}$ on $X$ satisfies the assumptions (i),(ii),(iii) of section 2 . 
We have the inclusions $U(\mathfrak{g})_{n+1} \subseteq U(\mathfrak{g})_{n}$ and similarly for the algebras $U(\mathfrak{g})^{\mathbf{G}}$ and $U(\mathfrak{t})$. The maps $\varphi, \phi$ and $\psi$ are visibly compatible with these inclusions. We therefore obtain an algebra homomorphism

$$
\operatorname{res}_{u}: \widehat{\mathcal{U}_{n+1, K}^{\lambda}} \longrightarrow \widehat{\mathcal{U}_{n, K}^{\lambda}}
$$

which fits into the commutative diagram

$$
\begin{aligned}
& \widehat{\mathcal{U}_{n+1, K}^{\lambda}} \stackrel{\widehat{\varphi_{n+1, K}^{\lambda}}}{\longrightarrow} \widehat{\mathcal{D}_{n+1, K}^{\lambda}} \\
& \widehat{\mathcal{U}}_{n, K}^{\downarrow^{\text {resu }}} \stackrel{\widehat{\varphi}_{n, K}^{\lambda}}{\longrightarrow} \widehat{\mathcal{D}}_{n, K}^{\lambda} .
\end{aligned}
$$

\section{LOCALLY ANALYTiC REPRESENTATIONS}

5.1. We recall some definitions and results about distribution algebras of compact locally $L$-analytic groups ([52], [54]). We consider a locally $L$-analytic group $G$ and denote by $C^{a n}(G, K)$ the locally convex $K$-vector space of locally $L$-analytic functions on $G$ as defined in [52]. The strong dual

$$
D(G):=D(G, K):=C^{a n}(G, K)_{b}^{\prime}
$$

is the algebra of $K$-valued locally analytic distributions on $G$. The multiplication $\delta_{1} \cdot \delta_{2}$ of distributions $\delta_{1}, \delta_{2} \in D(G)$ is given by the convolution product

$$
\delta_{1} \cdot \delta_{2}(f)=\delta_{2}\left(h_{2} \mapsto \delta_{1}\left(h_{1} \mapsto f\left(h_{1} h_{2}\right)\right)\right) .
$$

Since $G$ is compact, $D(G)$ is a $K$-Fréchet algebra.

5.2. The universal enveloping algebra $U(\mathfrak{g})$ of the Lie algebra $\mathfrak{g}:=\operatorname{Lie}(G)$ of $G$ acts naturally on $C^{a n}(G, K)$. On elements $\mathfrak{x} \in \mathfrak{g}$ this action is given by

$$
(\mathfrak{x} f)(h)=\left.\frac{d}{d t}\left(t \mapsto f\left(\exp _{G}(-t \mathfrak{x}) h\right)\right)\right|_{t=0}
$$

where $\exp _{G}: \mathfrak{g}-->G$ denotes the exponential map of $G$, defined in a small neighbourhood of 0 in $\mathfrak{g}$. This gives rise to an embedding of $U(\mathfrak{g})_{K}:=U(\mathfrak{g}) \otimes_{L} K$ into $D(G)$ via

$$
U(\mathfrak{g})_{K} \hookrightarrow D(G), \mathfrak{x} \mapsto(f \mapsto(\dot{\mathfrak{x}} f)(1)) .
$$

Here $\mathfrak{x} \mapsto \dot{\mathfrak{x}}$ is the unique anti-automorphism of the $K$-algebra $U(\mathfrak{g})_{K}$ which induces multiplication by -1 on $\mathfrak{g}$.

5.3. We will occasionally write $G_{0}$ for the same group $G$ but with the locally $\mathbb{Q}_{p}$-analytic structure induced by restriction of scalars. We point out that there is a canonical isomorphism of $\mathbb{Q}_{p}$-Lie algebras $\operatorname{Lie}(G) \simeq \operatorname{Lie}\left(G_{0}\right)$ ([12], 5.14.5) which we will use to identify 
both $\mathbb{Q}_{p}$-algebras from now on. The inclusion of locally $L$-analytic functions into locally $\mathbb{Q}_{p}$-analytic functions on $G$ gives rise to a quotient map of topological algebras $D\left(G_{0}\right) \rightarrow D(G)$ whose kernel ideal is generated by the kernel of the natural map

$$
L \otimes_{\mathbb{Q}_{p}} \operatorname{Lie}\left(G_{0}\right) \longrightarrow \operatorname{Lie}(G), a \otimes \mathfrak{x} \mapsto a \mathfrak{x}
$$

(46], Lemma 5.1).

5.5. We will now specialize to uniform pro- $p$ groups. We refer to [18 for an extensive study of this important class of locally $\mathbb{Q}_{p}$-analytic groups. We assume for the rest of this section that $p \neq 2$. So let $G$ be a uniform pro- $p$ group of dimension $d:=\operatorname{dim}_{\mathbb{Q}_{p}} G$. Let $h_{1}, \ldots, h_{d}$ be a set of topological generators. In particular, any element $g \in G$ can uniquely be written as $g=h_{1}^{\lambda_{1}} \cdots h_{d}^{\lambda_{d}}$ with $\lambda_{1}, \ldots, \lambda_{d} \in \mathbb{Z}_{p}$. According to [loc.cit.], Thm. 4.30 the operations

$$
\begin{aligned}
\lambda \cdot x & =x^{\lambda} \\
x+y & =\lim _{i \rightarrow \infty}\left(x^{p^{i}} y^{p^{i}}\right)^{p^{-i}} \\
{[x, y] } & =\lim _{i \rightarrow \infty}\left(x^{-p^{i}} y^{-p^{i}} x^{p^{i}} y^{p^{i}}\right)^{p^{-2 i}}
\end{aligned}
$$

for $x, y \in G$ and $\lambda \in \mathbb{Z}_{p}$ define on the set $G$ the structure of a Lie algebra $L_{G}$ over $\mathbb{Z}_{p}$. This Lie algebra is powerful in the sense that it is a free $\mathbb{Z}_{p}$-module (of finite rank $d$ ) and satisfies $\left[L_{G}, L_{G}\right] \subseteq p L_{G}$.

The logarithm map $\log _{G}$ of the Lie group $G$ induces an injective homomorphism of $\mathbb{Z}_{p}$-Lie algebras $L_{G} \hookrightarrow \operatorname{Lie}(G)$ ([18], sect. 4.5/9.4). We will therefore view $L_{G}$ as a distinguished $\mathbb{Z}_{p}$-lattice in the $\mathbb{Q}_{p}$-Lie algebra $\operatorname{Lie}(G)$.

5.6. Now let $G$ be a compact locally $L$-analytic group of $\operatorname{dimension} d=\operatorname{dim}_{L} G$. Following [46], 42] we call $G L$-uniform if

(1) $G$ is uniform pro- $p$,

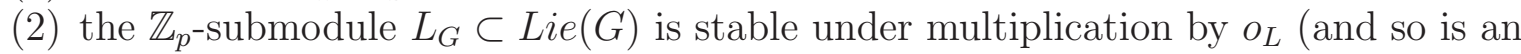
$o_{L}$-submodule of $\left.\operatorname{Lie}(G)\right)$.

We remark that any compact locally $L$-analytic group $G$ has a basis of neighbourhoods of $1 \in G$ consisting of open normal subgroups which are $L$-uniform ([46], Cor.4.4).

Let in the following $G$ be a locally $L$-analytic group of dimension $d$ which is $L$-uniform. Then $L_{G}$ is a free $o_{L}$-module of rank $d$. Let $\mathfrak{x}_{1}, \ldots \mathfrak{x}_{d}$ be an $o_{L}$-basis of $L_{G}$ and let $v_{1}, \ldots, v_{\left[L: \mathbb{Q}_{p}\right]}$ be a $\mathbb{Z}_{p}$-basis of $o_{L}$ with the additional property $v_{1}=1$. Then the elements

$$
h_{i j}:=\exp _{G}\left(v_{i} \mathfrak{x}_{j}\right)
$$

are a set of topological generators of $G$ of cardinality $\left[L: \mathbb{Q}_{p}\right] d=\operatorname{dim}_{\mathbb{Q}_{p}} G_{0}$.

5.7. The underlying $K$-vector space of $D\left(G_{0}\right)$ admits the following description. Fix an ordering of the generators $h_{i j}$. Let $b_{i j}:=h_{i j}-1 \in \mathbb{Z}[G]$ and $\mathbf{b}^{\alpha}:=\prod_{i j} b_{i j}^{\alpha_{i j}} \in \mathbb{Z}[G]$ (in the 
ON LOCALLY ANALYTIC BEILINSON-BERNSTEIN LOCALIZATION AND THE CANONICAL DIMENSIOQXY

given ordering) for $\alpha \in \mathbb{N}_{0}^{\left[L: \mathbb{Q}_{p}\right] d}$. Then $D\left(G_{0}\right)$ equals the set of all convergent series

$$
\lambda=\sum_{\alpha \in \mathbb{N}_{0}^{\left[L: \mathbb{Q}_{p}\right] d}} d_{\alpha} \mathbf{b}^{\alpha}
$$

with $d_{\alpha} \in K$ such that the set $\left\{\left|d_{\alpha}\right| r^{|\alpha|}\right\}_{\alpha}$ is bounded for all real numbers $0<r<1$. The inclusion $\operatorname{Lie}\left(G_{0}\right) \subset D\left(G_{0}\right)$ is given on basis elements by

$$
v_{i} \mathfrak{x}_{j} \mapsto \log \left(1+b_{i j}\right)
$$

where $\log (1+X)=X-X^{2} / 2+X^{3} / 3-\cdots$ is the usual logarithm series. The family of norms $\|.\|_{r}, 0<r<1$ where

$$
\|\lambda\|_{r}:=\sup _{\alpha}\left|d_{\alpha}\right| r^{|\alpha|}
$$

and their quotient norms define the Fréchet topologies on $D\left(G_{0}\right)$ respectively $D(G)$. The norms belonging to the interval $p^{-1} \leq r<1$ where $r \in p^{\mathbb{Q}}$ are multiplicative. In this case the corresponding norm completions $D_{r}\left(G_{0}\right)$ and $D_{r}(G)$ of $D\left(G_{0}\right)$ and $D(G)$ respectively are $K$-Banach algebras. Furthermore, we emphasize that the norms in this case do not depend on the choice of minimal set of topological generators for the group $G$ ([54], discussion after Thm. 4.10).

5.9. Let $r \in p^{\mathbb{Q}}$ such that $p^{-1} \leq r<1$. There is the following norm filtration on $D_{r}\left(G_{0}\right)$ defined by the additive subgroups

$$
\begin{gathered}
F_{r}^{s} D_{r}\left(G_{0}\right):=\left\{\lambda \in D_{r}\left(G_{0}\right),\|\lambda\|_{r} \leq p^{-s}\right\}, \\
F_{r}^{s+} D_{r}\left(G_{0}\right):=\left\{\lambda \in D_{r}\left(G_{0}\right),\|\lambda\|_{r}<p^{-s}\right\}
\end{gathered}
$$

for $s \in \mathbb{R}$ with graded ring

$$
g r_{r}^{\bullet} D_{r}\left(G_{0}\right):=\oplus_{s \in \mathbb{R}} g r_{r}^{s} D_{r}\left(G_{0}\right)
$$

where $g r_{r}^{s} D_{r}\left(G_{0}\right):=F_{r}^{s} D_{r}\left(G_{0}\right) / F_{r}^{s+} D_{r}\left(G_{0}\right)$.

We may use the quotient norm of $\|.\|_{r}$ to define a norm filtration on $D_{r}(G)$ as above. It coincides with the quotient filtration of $F_{r}^{\bullet} D_{r}\left(G_{0}\right)$. We denote the associated graded ring by $g r_{r}^{\bullet} D_{r}(G)$.

Similarly, the absolute value on $K$ induces a filtration and a graded ring $g r K$ as above. The choice of uniformizer $\pi_{K}$ induces an isomorphism of $\operatorname{gr} K$ with the ring of Laurent polynomials in one variable over $k$. The rings $g r_{r}^{\bullet} D_{r}\left(G_{0}\right)$ and $g r_{r}^{\bullet} D_{r}(G)$ are naturally gr $K$-algebras.

Now let additionally $p^{-1}<r<1$. Then Thm. 4.5 of [54] shows that each $g r_{r}^{\bullet} D_{r}\left(G_{0}\right)$ equals a polynomial ring over $\operatorname{gr} K$ in $\left[L: \mathbb{Q}_{p}\right] d$ variables (the principal symbols of the $\left.b_{i j}\right)$. Hence any $g r_{r}^{\bullet} D_{r}(G)$ will be a commutative $g r K$-algebra.

5.10. Let $\left\{G^{p^{m}}\right\}_{m \geq 0}$ be the lower $p$-series of the uniform pro- $p$ group $G$, i.e.

$$
G^{p^{m}}:=\left\{g^{p^{m}}: g \in G\right\}
$$


for $m \geq 0$. Each $G^{p^{m}}$ is a characteristic open uniform pro- $p$ subgroup of $G$ of index $p^{\left[L: \mathbb{Q}_{p}\right] m \bar{d}}$. The set $\left\{G^{p^{m}}\right\}_{m}$ constitutes a neighbourhood basis of $1 \in G$. Because of $L_{G_{0}^{p^{m}}}=$ $p^{m} L_{G_{0}}$ each open subgroup $G^{p^{m}}$, endowed with its induced locally $L$-analytic structure, is $L$-uniform. By functoriality of $D(\cdot)([31], 1.1)$ we obtain a series of subalgebras

$$
D\left(G_{0}\right) \supset D\left(G_{0}^{p}\right) \supset D\left(G_{0}^{p^{2}}\right) \supset \ldots
$$

which yields, by passage to quotients, a series

$$
D(G) \supset D\left(G^{p}\right) \supset D\left(G^{p^{2}}\right) \supset \ldots
$$

of subalgebras of $D(G)$. For each $m \geq 0$ we may apply the above discussion to the group $G^{p^{m}}$ and obtain a family of norms $\|\cdot\|_{r}^{(m)}$ on $D\left(G_{0}^{p^{m}}\right)$ as well as quotient norms $q_{r}^{m}$ on $D\left(G^{p^{m}}\right)$. Of course, these norms again induce filtrations and graded rings as explained above whenever $r \in p^{\mathbb{Q}}$ with $p^{-1} \leq r<1$.

To ease notation we put for $m \geq 0$ from now on

$$
r_{m}:=\sqrt[p]{1 / p}
$$

In particular, $r_{0}=1 / p$.

Lemma 5.11. The ring extension $D\left(G^{p^{m}}\right) \subset D(G)$ completes to a ring extension

$$
D_{r_{0}}\left(G^{p^{m}}\right) \subset D_{r_{m}}(G)
$$

and $D_{r_{m}}(G)$ is a finite and free (left or right) module over $D_{r_{0}}\left(G^{p^{m}}\right)$ on a basis any system of coset representatives for the finite group $G / G^{p^{m}}$.

Proof. This is a slight generalization of [46], Lemma 7.4 using the fact that the norm $\|.\|_{r_{m}}^{(0)}$ on $D\left(G_{0}\right)$ restricts to $\|.\|_{r_{0}}^{(m)}$ on the subring $D\left(G_{0}^{p^{m}}\right) \subset D\left(G_{0}\right)$. This latter fact is itself a mild extension of [loc.cit.], Prop. 6.2 which follows with the same proof given there.

5.12. Completely similar to the standard example of the group ring ([43]) the $\operatorname{ring} D(G)$ is a crossed product of the ring $S:=D\left(G^{p^{m}}\right)$ by the group $H:=G / G^{p^{m}}$ with action and twisting given as follows. Fix a set of representatives $\bar{H} \subset G$ for the group $G / G^{p^{m}}$ and view it in $D(G)^{\times}$via the map $\delta$ (associated Dirac distribution). Then

$$
\sigma(h)(s)=\delta_{\bar{h}}^{-1} s \delta_{\bar{h}}
$$

for $s \in S$. On the other hand, given $h_{1}, h_{2} \in H$ we have

$$
\bar{h}_{1} \bar{h}_{2}=\overline{h_{1} h_{2}} \cdot \tau\left(h_{1}, h_{2}\right)
$$

with some uniquely determined $\tau\left(h_{1}, h_{2}\right) \in G^{p^{m}}$ and the resulting $\tau: H \times H \rightarrow G^{p^{m}} \subseteq S^{\times}$ equals the twisting.

As we have pointed out the norm $\|.\|_{r_{0}}^{(m)}$ on $S=D\left(G^{p^{m}}\right)$ is independent of the choice of topological generators for the group $G^{p^{m}}$. This means that for $g \in G$ the conjugation automorphism on $S=D\left(G^{p^{m}}\right)$ given by $x \mapsto \delta_{g}^{-1} x \delta_{g}$ is an isometry. Thus, $\sigma$ extends to an 
action $H \rightarrow \operatorname{Out}\left(D_{r_{0}}\left(G^{p^{m}}\right)\right)$. Likewise $\tau$ extends to a 2-cocycle with values in $D_{r_{0}}\left(G^{p^{m}}\right)^{\times}$ and we may form the crossed product

$$
D_{r_{0}}\left(G^{p^{m}}\right) *_{\sigma, \tau} H
$$

Corollary 5.13. There is a natural K-algebra isomorphism

$$
D_{r_{0}}\left(G^{p^{m}}\right) *_{\sigma, \tau} G / G^{p^{m}} \stackrel{\cong}{\longrightarrow} D_{r_{m}}(G) \text {. }
$$

Proof. The preceding lemma gives a $K$-algebra homomorphism $D_{r_{0}}\left(G^{p^{m}}\right) *_{\sigma, \tau} G / G^{p^{m}} \rightarrow$ $D_{r_{m}}(G)$ induced from the inclusion $D_{r_{0}}\left(G^{p^{m}}\right) \subset D_{r_{m}}(G)$ which is bijective.

Remark: In the case $L=\mathbb{Q}_{p}$ this responds to an issue raised in [4], 1.5.

5.14. We fix $m \geq 1$ and consider the filtration on the Banach algebra $D_{r_{0}}\left(G^{p^{m}}\right)$ induced by the quotient norm $q_{r_{0}}^{m}$ from 5.10. The $\left[L: \mathbb{Q}_{p}\right] d$ elements $h_{i j}^{p^{m}}$ are a minimal ordered set of topological generators for the group $G^{p^{m}}$. Define the $d$ elements $c_{j}:=h_{1 j}^{p^{m}}-1$ and let $\mathbf{c}^{\beta}=\prod_{j} c_{j}^{\beta_{j}}$ for $\beta \in \mathbb{N}^{d}$.

Lemma 5.15. Let $m \geq 1$. The graded ring $\mathrm{gr}_{r_{0}}^{\bullet} D_{r_{0}}\left(G^{p^{m}}\right)$ equals a polynomial algebra over gr $K$ in the principal symbols of the $d$ elements $c_{j}$. The K-vector space underlying $D_{r_{0}}\left(G^{p^{m}}\right)$ is given by all series

$$
\lambda=\sum_{\beta \in \mathbb{N}_{0}^{d}} d_{\beta} \mathbf{c}^{\beta}
$$

with $\left|d_{\beta}\right| r_{0}{ }^{|\beta|} \rightarrow 0$ for $|\beta| \rightarrow \infty$. Moreover, the norm $q_{r_{0}}^{m}(\lambda)$ may be computed as

$$
q_{r_{0}}^{m}(\lambda)=\sup _{\beta} q_{r_{0}}^{m}\left(d_{\beta} \mathbf{c}^{\beta}\right)=\sup _{\beta}\left|d_{\beta}\right| r_{0}|\beta| .
$$

Proof. We have already remarked (proof of lemma 5.11) that $\|.\|_{r_{m}}^{(0)}$ restricts to $\|.\|_{r_{0}}^{(m)}$ on the subring $D\left(G_{0}^{p^{m}}\right) \subset D\left(G_{0}\right)$ from which we obtain an inclusion of rings

$$
g r_{r_{0}} D_{r_{0}}\left(G_{0}^{p^{m}}\right) \subset g r_{r_{m}} D_{r_{m}}\left(G_{0}\right)
$$

by left exactness of the functor $g r$. Since $m \geq 1$ we have $p^{-1}<r_{m}$. Thus the right-hand side is a commutative ring. It follows that $g r_{r_{0}} D_{r_{0}}\left(G_{0}^{p^{m}}\right)$ is a polynomial algebra over $g r K$ in the $n d$ principal symbols of the $h_{i j}^{p^{m}}-1$. Arguing as in [46], Prop. 5.6/9 the quotient $g r_{r_{0}} D_{r_{0}}\left(G^{p^{m}}\right)$ equals a polynomial algebra in the principal symbols of the $c_{j}$ and the remaining statements follow from this.

Remark: The result generally does not hold for $m=0$ : let $L=\mathbb{Q}_{p}$ and

$$
\mathbb{Z}_{p}[[G]]=\lim _{N} \mathbb{Z}_{p}[G / N]
$$

be the completed group ring of the profinite group $G$. Here, $N$ varies over the open normal subgroups of $G$. There is an embedding $\mathbb{Z}_{p}[[G]] \hookrightarrow D_{r_{0}}(G)$. By one of the main 
theorems of M. Lazard's work on p-adic analytic groups ([33], Thm. III.2.3.3) the algebra $g r_{r_{0}} \mathbb{Z}_{p}[[G]]$ equals the universal enveloping algebra of a certain Lie algebra associated to the group $G$ which, generally, is non-abelian.

Still assuming $m \geq 1$ the preceding discussion shows that the $o_{K^{-}}$-submodule $F_{r_{0}}^{0} D_{r_{0}}\left(G^{p^{m}}\right)$ of $D_{r_{0}}\left(G^{p^{m}}\right)$ consists of all series

$$
\lambda=\sum_{\beta \in \mathbb{N}_{0}^{d}} e_{\beta}\left(c_{1} / p\right)^{\beta_{1}} \cdots\left(c_{d} / p\right)^{\beta_{d}}
$$

with $\left|e_{\beta}\right| \leq 1$ and $\left|e_{\beta}\right| \rightarrow 0$ for $|\beta| \rightarrow \infty$. Mapping such a series to $\sum_{\beta}\left(e_{\beta} \bmod \pi_{K}\right) u_{1}^{\beta_{1}} \cdots u_{d}^{\beta_{d}}$ induces an isomorphism of $k$-algebras between

$$
g r_{r_{0}}^{0} D_{r_{0}}\left(G^{p^{m}}\right)=F_{r_{0}}^{0} D_{r_{0}}\left(G^{p^{m}}\right) / \pi_{K} F_{r_{0}}^{0} D_{r_{0}}\left(G^{p^{m}}\right)
$$

and the polynomial algebra over $k$ in the variables $u_{1}, \ldots, u_{d}$. We see that the $o_{K}$-subalgebra $F_{r_{0}}^{0} D_{r_{0}}\left(G^{p^{m}}\right)$ is a $\pi_{K}$-adically separated and complete lattice in the $K$-algebra $D_{r_{0}}\left(G^{p^{m}}\right)$. Moreover, the $\pi_{K}$-adic reduction of this lattice is endowed with a positive and hence complete split $\mathbb{Z}$-filtration given by total degree with respect to the variables $u_{j}$. We conclude that $D_{r_{0}}\left(G^{p^{m}}\right)$ is a complete doubly filtered $K$-algebra in the sense of [4, Def. 3.1 with

$$
\operatorname{Gr}\left(D_{r_{0}}\left(G^{p^{m}}\right)\right):=\operatorname{gr}\left(g r_{r_{0}}^{0} D_{r_{0}}\left(G^{p^{m}}\right)\right) \simeq k\left[u_{1}, \ldots, u_{d}\right] .
$$

5.17. Let $G$ be a pro- $p$ group which is $L$-uniform. The projective system of noetherian $K$-Banach algebras $D_{r_{m}}(G)$ for $m \geq 1$ defines the so-called Fréchet-Stein structure of $D(G)$. Without recalling the precise definition from [54 this essentially means that the natural map

$$
D(G) \stackrel{\cong}{\lim _{m \geq 1}} D_{r_{m}}(G)
$$

is an isomorphism of topological $K$-algebras (where the right-hand side is endowed with the projective limit topology) and that the transition maps in the projective system are flat ring homomorphisms. This structure gives rise to a well-behaved abelian full subcategory $\mathscr{C}_{G}$ of the (left) $D(G)$-modules, the coadmissible modules. By definition, an abstract $D(G)$-module $M$ is coadmissible if

(i) $M_{m}:=D_{r_{m}}(G) \otimes_{D(G)} M$ is finitely generated over $D_{r_{m}}(G)$ for all $m \geq 1$;

(ii) the natural map $M \stackrel{\cong}{\longleftarrow} \lim _{m} M_{m}$ is an isomorphism.

The system $\left\{M_{m}\right\}_{m}$ is sometimes called the coherent sheaf associated to $M$. In fact, the projective limit functor induces an equivalence of categories between the projective systems $\left(M_{m}\right)_{m}$ of finitely generated $D_{r_{m}}(G)$-modules $M_{m}$ with the property

$$
D_{r_{m}}(G) \otimes_{D_{r_{m+1}}(G)} M_{m+1} \stackrel{\cong}{\longrightarrow} M_{m}
$$


and $\mathscr{C}_{G}$. To give an example, any $D(G)$-module which is finitely presented as $D(G)$-module is coadmissible.

Remarks: The theory of Fréchet-Stein algebras is modelled according to the example $G=\mathbb{Z}_{p}$, the additive group of $p$-adic integers. In this case, the Fourier isomorphism of $\mathrm{Y}$. Amice ([1]) identifies $D\left(\mathbb{Z}_{p}\right)$ with the ring of holomorphic functions on the rigid analytic open unit disc over $K$. The latter is a quasi-Stein space in the sense of R. Kiehl ([30]) and the Fourier isomorphism identifies the category of coadmissible modules with the coherent module sheaves on the disc.

5.18. For the definition of an admissible locally analytic representation as well as the foundations of the theory of such representations we refer to [54. However, we at least recall ([loc.cit.], Thm. 6.3) that the abelian category $\operatorname{Rep}(G)$ of admissible locally analytic $G$-representations over $K$ is (essentially by definition of admissibility) anti-equivalent to $\mathscr{C}_{G}$,

$$
\operatorname{Rep}(G) \stackrel{\cong}{\longrightarrow} \mathscr{C}_{G}
$$

The functor is given by sending a representation $V$ to its so-called strong dual $M:=V_{b}^{\prime}$. As a locally convex $K$-vector space $V_{b}^{\prime}$ equals the continuous dual of the locally convex vector space $V$ endowed with a certain strong topology (see [49] for all terminology from non-archimedean functional analysis). The contragredient $G$-representation on $V_{b}^{\prime}$ extends naturally to a $D(G)$-module structure giving the coadmissible module.

\section{An APPLICATION OF AN ISOMORPHISM OF LAZARD}

In this section we assume $p \neq 2$.

6.1. Let $G$ be a uniform pro- $p$ group. Its integral $\mathbb{Z}_{p}$-Lie algebra $L_{G}$ was first mentioned by M. Lazard ([32], Ex. III. 2.1.10). We require in the following some information on the relation of $L_{G}$ with the group ring $\mathbb{Z}_{p}[G]$ of $G$ and we therefore recall parts of Lazard's notions and results. We, however, do not recall the standard definitions of filtrations, valuations etc. (cf. loc.cit., I.1).

To start with, the group ring $\mathbb{Z}_{p}[G]$ has a canonical filtration defined as the lower bound of all filtrations $\omega$ of the $\mathbb{Z}_{p}$-algebra $\mathbb{Z}_{p}[G]$ such that

$$
\omega(g-1) \geq \omega(g)
$$

for all $g \in G$ (loc.cit., III.2.3.1.2). Since $G$ is a uniform pro- $p$ group $\omega$ is in fact a valuation of the $\mathbb{Z}_{p}$-module $\mathbb{Z}_{p}[G]$ which allows to define its so-called saturation Sat $\mathbb{Z}_{p}[G]$ (loc.cit., I.2.2.11). The latter equals the completion of

$$
\left\{y \in \mathbb{Q}_{p}[G] \mid \tilde{\omega}(y) \geq 0\right\}
$$

in the topology defined by $\tilde{\omega}$ and is an associative algebra containing $\mathbb{Z}_{p}[G]$. Note here that the valuation $\omega$ on $\mathbb{Z}_{p}[G]$ is extended to a map $\tilde{\omega}$ on $\mathbb{Q}_{p}[G]=\mathbb{Q}_{p} \otimes_{\mathbb{Z}_{p}} \mathbb{Z}_{p}[G]$ by

$$
\tilde{\omega}\left(\lambda^{-1} \otimes m\right):=\omega(m)-v_{p}(\lambda)
$$


where $v_{p}($.$) equals the p$-adic valuation on $\mathbb{Z}_{p}$.

On the other hand, the Lie algebra $L_{G}$ is a finite free $\mathbb{Z}_{p}$-module. Fixing a basis and using the valuation $v_{p}$ on coefficients it is a valued $\mathbb{Z}_{p}$-module. This valuation induces a valuation on the universal enveloping algebra $U\left(L_{G}\right)$ (loc.cit., IV. 2.2.1) and the corresponding saturation Sat $U\left(L_{G}\right)$ does not depend on the choice of valuation of $L_{G}$. Put $\mathfrak{h}:=\frac{1}{p} L_{G}$. This is a $\mathbb{Z}_{p}$-Lie algebra since $L_{G}$ is powerful. Let

$$
\widehat{U(\mathfrak{h})}=\left({\underset{\leftarrow}{i}}_{\lim } U(\mathfrak{h}) / p^{i} U(\mathfrak{h})\right)
$$

be the $p$-adic completion of $U(\mathfrak{h})$. The inclusion $L_{G} \subset \mathfrak{h}$ induces an isomorphism of Sat $U\left(L_{G}\right)$ with $\widehat{U(\mathfrak{h})}$ which, combined with the isomorphism loc.cit., IV. 3.2.5, gives an isomorphism

$$
\mathcal{L}_{G}: \widehat{U(\mathfrak{h})} \stackrel{\cong}{\longrightarrow} \text { Sat } \mathbb{Z}_{p}[G] .
$$

By loc.cit., Cor. 3.2.4 it is given on elements $g \in L_{G}$ via $\mathcal{L}_{G}(g):=\log (g)$ where

$$
\log (X)=(X-1)-(X-1)^{2} / 2+(X-3)^{3} / 3-\cdots
$$

is the usual logarithm power series (loc.cit., III.1.1.5.2).

Remark: A concise account of the integral Lazard Lie algebra and its various relations to the group ring can also be found in [24].

Let

$$
\widehat{U(\mathfrak{h})_{\mathbb{Q}_{p}}}:=\widehat{U(\mathfrak{h})} \otimes_{\mathbb{Z}_{p}} \mathbb{Q}_{p} .
$$

Proposition 6.3. The map $\mathcal{L}_{G}$ and the inclusion $\mathbb{Z}_{p}[G] \subset D\left(G, \mathbb{Q}_{p}\right)$ induce an isomorphism of $\mathbb{Q}_{p}$-Banach algebras

$$
\mathcal{L}_{G}: \widehat{U(\mathfrak{h})_{\mathbb{Q}_{p}}} \stackrel{\cong}{\longrightarrow} D_{r_{0}}\left(G, \mathbb{Q}_{p}\right) .
$$

compatible with the canonical maps $\mathfrak{h} \hookrightarrow \operatorname{Lie}(G) \hookrightarrow D\left(G, \mathbb{Q}_{p}\right)$.

Proof. This reformulation of the Lazard isomorphism is essentially [2], Thm. 5.1.4 (compare also [4], Thm. 10.4 and the remarks 10.5). Indeed, according to loc.cit. the map $\mathcal{L}_{G}$ induces an isomorphism between $\widehat{U(\mathfrak{h})_{\mathbb{Q}_{p}}}$ and the completion of $\mathbb{Q}_{p} \Lambda_{G}:=\mathbb{Q}_{p} \otimes_{\mathbb{Z}_{p}} \mathbb{Z}_{p}[[G]]$ with respect to a certain norm on $\mathbb{Q}_{p} \Lambda_{G}$ constructed in [18], chap. 7. By the explicit formulae given in loc.cit. this norm equals the restriction of $\|.\|_{r_{0}}$ to $\mathbb{Q}_{p} \Lambda_{G}$. But the latter space is norm-dense in $D_{r_{0}}\left(G, \mathbb{Q}_{p}\right)$ which gives the first claim. The compatibility with the inclusions $\mathfrak{h} \hookrightarrow \operatorname{Lie}(G) \hookrightarrow D\left(G, \mathbb{Q}_{p}\right)$ follows from (5.8).

Corollary 6.5. The inverse $\mathcal{L}_{G}^{-1}$ induces an injective group homomorphism

$$
\mathcal{L}_{G}^{-1}: G \hookrightarrow \widehat{U(\mathfrak{h})}^{\times} .
$$


ON LOCALLY ANALYTIC BEILINSON-BERNSTEIN LOCALIZATION AND THE CANONICAL DIMENSIOZX

Proof. The inclusion $\mathbb{Z}_{p}[G] \subset$ Sat $\mathbb{Z}_{p}[G]$ identifies $G$ with a subgroup of units of the ring Sat $\mathbb{Z}_{p}[G]$.

6.6. We now turn back to the setting of section 3. Recall the $o_{L}$-Lie algebra $\mathfrak{g}$ of the connected split reductive group scheme $\mathbf{G}$. Let $\mathfrak{g}_{0}$ equal $\mathfrak{g}$ but viewed over $\mathbb{Z}_{p}$ and recall that $e$ is the ramification index of $L / \mathbb{Q}_{p}$. We assume that we are given a $L$-uniform pro- $p$ group $G$ that satisfies the following hypothesis relative to $\mathbf{G}$ :

$$
\text { (HYP) } \quad L_{G}=\pi_{L}^{k} \mathfrak{g}_{0}
$$

as Lie algebras over $\mathbb{Z}_{p}$ for a natural number $k \geq 1$. We additionally fix a number $m \geq 1$. In the following we are studying the $\mathbb{Z}_{p}$-Lie algebra

$$
\mathfrak{h}:=\frac{1}{p} L_{G^{p^{m}}}=\frac{1}{p} p^{m} L_{G}=p^{m} \frac{1}{p} L_{G}=\pi_{L}^{(m-1) e+k} \mathfrak{g}_{0} .
$$

Abbreviating $n:=(m-1) e+k \geq 1$, the Lazard isomorphism applied to $G^{p^{m}}$ is an isomorphism

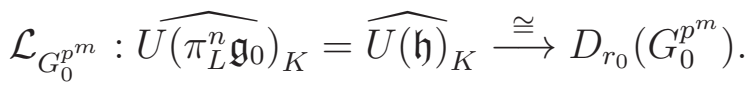

Fix an ordered $o_{L}$-basis $\mathfrak{y}_{1}, \ldots, \mathfrak{y}_{d}$ of $\mathfrak{g}$ and a $\mathbb{Z}_{p}$-basis $v_{1}, \ldots, v_{\left[L: \mathbb{Q}_{p}\right]}$ of $o_{L}$ with $v_{1}=1$. Given $\alpha \in \mathbb{N}_{0}^{\left[L: \mathbb{Q}_{p}\right] d}$, we form the elements

$$
\mathfrak{Y}^{\alpha}:=\left(v_{1} \mathfrak{y}_{1}\right)^{\alpha_{11}} \cdots\left(v_{\left[L: \mathbb{Q}_{p}\right]} \mathfrak{y}_{d}\right)^{\left.\alpha_{[L: \mathbb{Q} p}\right] d}
$$

inside $U(\mathfrak{g})$. The $K$-Banach algebra $\widehat{U\left(\pi_{L}^{n} \mathfrak{g}_{0}\right)_{K}}=\widehat{U\left(\pi_{L}^{n} \mathfrak{g}_{0}\right)} \otimes_{\mathbb{Z}_{p}} K$ is given by all series

$$
\lambda:=\sum_{\alpha \in \mathbb{N}_{0}^{\left[L: \mathbb{Q}_{p}\right] d}} d_{\alpha} \mathfrak{Y}^{\alpha}
$$

where $d_{\alpha} \in K$ with $\left|d_{\alpha}\right| \cdot\left|\pi_{L}\right|^{-n \cdot|\alpha|} \rightarrow 0$ for $|\alpha| \rightarrow \infty$. It contains the kernel $J$ of the map

$$
\mathfrak{g}_{0} \otimes_{\mathbb{Z}_{p}} o_{L} \rightarrow \mathfrak{g}, \mathfrak{x} \otimes a \mapsto a \mathfrak{x} .
$$

If the set $J$ injects into some algebra we denote by $\langle J\rangle$ the two-sided ideal generated by $J$ in this algebra. Reasoning as in (the proof of) [47], Prop. 2.4 the quotient algebra $\widehat{U\left(\pi_{L}^{n} \mathfrak{g}_{0}\right)_{o_{L}}} /\langle J\rangle$ is given by the $\pi_{L}$-adically complete algebra of all series

$$
\lambda:=\sum_{\beta \in \mathbb{N}_{0}^{d}} e_{\beta} \mathfrak{Y}^{\prime \beta}
$$

where $e_{\beta} \in o_{L}$ with $\left|e_{\beta}\right| \cdot\left|\pi_{L}\right|^{-n \cdot|\beta|} \rightarrow 0$ for $|\beta| \rightarrow \infty$ and $\mathfrak{Y}^{\prime \beta}:=\mathfrak{y}_{1}^{\beta_{1}} \cdots \mathfrak{y}_{d}^{\beta_{d}}$. It therefore coincides with $\widehat{U\left(\pi_{L}^{n} \mathfrak{g}\right)}=\widehat{U(\mathfrak{g})_{n}}$. Now taking into account the discussion preceding the map (5.4), the Lazard isomorphism (6.8) factores into an isomorphism

$$
\left.\mathcal{L}_{G^{p^{m}}}: \widehat{U(\mathfrak{g})_{n, K}}=\widehat{U(\mathfrak{g})_{n}} \otimes_{o_{L}} K=\widehat{U\left(\pi_{L}^{n} \mathfrak{g}_{0}\right.}\right)_{K} /\langle J\rangle \stackrel{\cong}{\longrightarrow} D_{r_{0}}\left(G_{0}^{p^{m}}\right) /\langle J\rangle=D_{r_{0}}\left(G^{p^{m}}\right)
$$


and the inverse $\mathcal{L}_{G^{p^{m}}}^{-1}$ yields an injective group homomorphism $G^{p^{m}} \hookrightarrow{\widehat{U(\mathfrak{g})_{n}}}^{\times}$. For future reference we summarize this discussion in a proposition.

Proposition 6.10. Let $G$ be a L-uniform pro-p group such that $L_{G}=\pi_{L}^{k} \mathfrak{g}_{0}$ for some $k \geq 1$. Let $m \geq 1$ and put $n:=(m-1) e+k$. The Lazard isomorphism induces an algebra isomorphism

$$
\mathcal{L}_{G^{p}}: \widehat{U(\mathfrak{g})_{n, K}} \stackrel{\cong}{\longrightarrow} D_{r_{0}}\left(G^{p^{m}}\right)
$$

whose inverse yields an injective group homomorphism $G^{p^{m}} \hookrightarrow{\widehat{U(\mathfrak{g})_{n}}}^{\times}$.

6.11. Let us keep the assumptions on the group $G$ appearing in the preceding proposition. Let $Z\left(\mathfrak{g}_{K}\right)$ be the center of the ring $U\left(\mathfrak{g}_{K}\right)$ and let $\theta: Z\left(\mathfrak{g}_{K}\right) \rightarrow K$ be an algebra homomorphism. We suppose that $Z\left(\mathfrak{g}_{K}\right) \hookrightarrow D(G)$ has image in the center of the ring $D(G)$. For example, this is true whenever $G$ is an open subgroup in the group of rational points of a connected algebraic group over $L$ ([52], Prop. 3.7). We then have the central reduction

$$
D(G)_{\theta}:=D(G) \otimes_{Z\left(\mathfrak{g}_{K}\right), \theta} K
$$

and similarly for any $D_{r}(G)$. We remark that, since the ring $Z\left(\mathfrak{g}_{K}\right)$ is noetherian, the ideal of $D(G)$ (and $D_{r}(G)$ ) generated by $\operatorname{ker} \theta$ is finitely generated and hence closed ([54], remark before Prop. 3.6). It follows from loc.cit., Prop. 3.6 that

$$
D(G)_{\theta} \stackrel{\cong}{\longrightarrow} \lim _{r} D_{r}(G)_{\theta} \text {. }
$$

On the other hand, given a weight $\lambda \in \operatorname{Hom}_{o_{L}}\left(\pi_{L}^{n} \mathfrak{t}, o_{K}\right)$ we have the central reduction ${\widehat{\mathcal{U}_{n}^{\lambda}, K}}_{\text {of }} \widehat{\mathcal{U}}_{n, K}$ from section 4 . We suppose that $\theta$ is related to $\lambda_{K} \in \mathfrak{t}_{K}^{*}$ via the (untwisted) Harish-Chandra homomorphism

$$
\phi_{K}: Z\left(\mathfrak{g}_{K}\right) \stackrel{\cong}{\longrightarrow} S\left(\mathfrak{t}_{K}\right)^{W, \bullet}
$$

([17], Thm. 7.4.5). Here, $(W, \bullet)$ refers to the usual dot-action

$$
w \bullet \mu:=w(\mu+\rho)-\rho
$$

of the Weyl group $W$ on a weight $\mu \in \mathfrak{t}_{K}$. According to ([16], II.§6.1.5) and since $\operatorname{char}(L)=0$, we have $U(\mathfrak{g})^{\mathbf{G}} \otimes_{o_{L}} L=Z\left(\mathfrak{g}_{L}\right)$ which means that $\phi_{K}$ comes by base change via $o_{L} \rightarrow K$ from the homomorphism $\phi: U(\mathfrak{g})^{\mathbf{G}} \rightarrow S(\mathfrak{t})$ of section 4 . This justifies our notation.

Corollary 6.13. The isomorphism $\mathcal{L}_{G^{p}}$ of the preceding proposition factores into an isomorphism

$$
\widehat{\mathcal{U}_{n, K}^{\lambda}} \stackrel{\cong}{\longrightarrow} D_{r_{0}}\left(G^{p^{m}}\right)_{\theta}
$$


Proof. Let $R:=\left(U(\mathfrak{g})^{\mathbf{G}}\right)_{n}$. By [4], Lemma 6.5 we have the canonical isomorphism

$$
\widehat{\mathcal{U}_{n, K}^{\lambda}}=\widehat{U(\mathfrak{g})_{n}} \otimes_{\hat{R}_{K}, \lambda} K \quad(*)
$$

where the completions are taken, as always, with respect to $p$-adic topologies. We claim that the induced topology on $R=\left(U(\mathfrak{g})^{\mathbf{G}}\right)_{n} \subset U(\mathfrak{g})_{n}$ is again the $p$-adic topology. Indeed, since $\mathbf{G}$ acts $o_{L}$-linearly on $U(\mathfrak{g})$ via its adjoint action, this is obvious for $n=0$. The general case $n>0$ is reduced to this case when we can show that the natural map

$$
\left(U(\mathfrak{g})^{\mathbf{G}}\right)_{n} \hookrightarrow\left(U(\mathfrak{g})_{n}\right)^{\mathbf{G}}
$$

is onto. Let $x \in U(\mathfrak{g})_{n}$ be $\mathbf{G}$-invariant. Since the deformed $P B W$-filtration on $U(\mathfrak{g})_{n}$ is still a split filtration and since $\mathbf{G}$ necessarily acts by strictly filtered isomorphisms we may assume that $x \in \pi_{L}^{i n} F_{i} U(\mathfrak{g})$ for some $i \geq 0$. But then

$$
x \in \pi_{L}^{i n}\left(F_{i} U(\mathfrak{g})\right)^{\mathbf{G}}=\pi_{L}^{i n} F_{i}\left(U(\mathfrak{g})^{\mathbf{G}}\right) \in\left(U(\mathfrak{g})^{\mathbf{G}}\right)_{n} .
$$

By the claim the $p$-adic completion $\hat{R}$ equals the closure of $R$ inside $\widehat{U(\mathfrak{g})_{n}}$. This means that $\hat{R}_{K}$ equals the closure $\overline{R_{K}}$ of $R_{K}=Z\left(\mathfrak{g}_{K}\right)$ inside $\widehat{U(\mathfrak{g})_{n}}, K$. So we deduce from (*) and the preceding proposition the isomorphism

$$
\widehat{\mathcal{U}_{n, K}^{\lambda}} \stackrel{\cong}{\longrightarrow} D_{r_{0}}\left(G^{p^{m}}\right) \otimes_{\overline{R_{K}}, \lambda} K=D_{r_{0}}\left(G^{p^{m}}\right) \otimes_{R_{K}, \lambda} K=D_{r_{0}}\left(G^{p^{m}}\right)_{\theta}
$$

which proves the assertion.

In the next section we will apply these results in the case where $G$ equals a certain principal congruence subgroup in the rational points of the group scheme $\mathbf{G}$.

\section{Congruence Kernels in SEMisimple $p$-ADiC Groups}

We assume in this section that $p \neq 2$.

7.1. We return to the setting of section 4. Consider the group $\mathbf{G}\left(o_{L}\right)$ of $o_{L}$-valued points of the split reductive group scheme $\mathbf{G}$. For every $k \geq 1$ we have in $\mathbf{G}\left(o_{L}\right)$ the normal congruence subgroup

$$
G\left(\pi_{L}^{k}\right):=\operatorname{ker}\left(\mathbf{G}\left(o_{L}\right) \longrightarrow \mathbf{G}\left(o_{L} / \pi_{L}^{k} o_{L}\right)\right) .
$$

Lemma 7.2. Let $k \geq e$. The locally L-analytic group $G\left(\pi_{L}^{k}\right)$ is L-uniform. The corresponding $o_{L}$-lattice in $\operatorname{Lie}\left(G\left(\pi_{L}^{k}\right)\right)=\mathfrak{g} \otimes_{o_{L}} L$ is given by $\pi_{L}^{k} \mathfrak{g}$.

Proof. The construction in [18], §13. Ex. 11 extends from the split semisimple case to the case of G. In particular, $G\left(\pi_{L}^{k}\right)$ is an $o_{L}$-standard group in the sense of loc.cit. or [13], III. $\$ 7.3$ and the corresponding $o_{L}$-lattice -mapping onto $G\left(\pi_{L}^{k}\right)$ via the exponential mapis given by $\pi_{L}^{k} \mathfrak{g}$. Since $k \geq e$, the $\mathbb{Z}_{p}$-Lie algebra $\pi_{L}^{k} \mathfrak{g}_{0}=p\left(\pi_{L}^{k-e} \mathfrak{g}_{0}\right)$ is powerful (recall $p \neq 2$ ) and therefore $G\left(\pi_{L}^{k}\right)_{0}$ is a uniform pro- $p$ group (cf. [18], Thm. 9.8).

According to the lemma we have $L_{G\left(\pi_{L}^{k}\right)}=\pi_{L}^{k} \mathfrak{g}_{0}$ for all $k \geq e$. Hence the hypothesis (HYP) from (6.6) is satisfied. Applying Prop. 6.10 yields the following proposition. 
Proposition 7.3. Let $G:=G\left(\pi_{L}^{k}\right)$ with $k \geq e$. Let $m \geq 1$ and put $n:=(m-1) e+k$. There is a canonical algebra isomorphism

$$
\mathcal{L}_{G^{p^{m}}}: \widehat{U(\mathfrak{g})_{n, K}} \stackrel{\cong}{\longrightarrow} D_{r_{0}}\left(G^{p^{m}}\right)
$$

whose inverse yields an injective group homomorphism $G^{p^{m}} \hookrightarrow{\widehat{U(\mathfrak{g})_{n}}}^{\times}$.

7.4. In the following we let $G=G\left(\pi_{L}^{k}\right)$ be a congruence subgroup for $k \geq e$. We have the finite group

$$
H_{m}:=G / G^{p^{m}} .
$$

We define inductively a set $\bar{H}_{m}$ of representatives for $H_{m}$ in $G$ containing $1 \in G$ with the property

$$
\bar{H}_{m+1}=\left\{\bar{g} \bar{h}: \bar{g} \in \bar{H}_{m, m+1}, \bar{h} \in \bar{H}_{m}\right\}
$$

where $\bar{H}_{m, m+1}$ is a set of representatives for the group $G^{p^{m}} / G^{p^{m+1}}$. Let

$$
s_{m}: H_{m} \longrightarrow G
$$

be the corresponding section of the canonical projection homomorphism $G \rightarrow H_{m}$. As usual we write $\bar{h}=s_{m}(h)$ for $h \in H_{m}$.

As in 5.12 we have a map $\tau^{\prime}: H_{m} \times H_{m} \rightarrow G^{p^{m}}$ by requiring

$$
\bar{h}_{1} \bar{h}_{2}=\overline{h_{1} h_{2}} \cdot \tau^{\prime}\left(h_{1}, h_{2}\right)
$$

in $G^{p^{m}}$ for $\bar{h}_{i} \in \bar{H}_{m}$ representatives of the classes $h_{i}$. As in the above proposition, we let from now on

$$
n:=n(m):=(m-1) e+k
$$

which is $\geq 1$. The group $G$ acts from the right via the adjoint representation $g \mapsto \operatorname{Ad}\left(g^{-1}\right)$ on $U(\mathfrak{g}) 3$ This action descends to the deformation $U(\mathfrak{g})_{n}$ and extends then to the $p$-adic completion $\widehat{U(\mathfrak{g})_{n}}$. Since $\widehat{U(\mathfrak{g})^{\mathbf{G}}}$ is fixed pointwise by this action we have an action on

$$
\widehat{\mathcal{U}_{n}^{\lambda}}=\widehat{U(\mathfrak{g})_{n}} \otimes_{\widehat{U(\mathfrak{g})^{\mathbf{G}}, \lambda}} O_{K}
$$

by letting $G$ act on the left factor. We therefore have a group homomorphism $G \rightarrow$ $\operatorname{Aut}\left(\widehat{\mathcal{U}_{n}^{\lambda}}\right)$ and, consequently, the map

$$
\sigma_{u}: H_{m} \stackrel{s_{m}}{\longrightarrow} G \longrightarrow \operatorname{Aut}\left(\widehat{\mathcal{U}_{n}^{\lambda}}\right) .
$$

On the other hand, there is the composite map

$$
\tau_{u}: H_{m} \times H_{m} \stackrel{\tau^{\prime}}{\longrightarrow} G^{p^{m}} \stackrel{\mathcal{L}_{G p}^{-1}}{\longrightarrow}{\widehat{U(\mathfrak{g})_{n}}}^{\times} \longrightarrow \widehat{\mathcal{U}}_{n}^{\lambda}
$$

where the final map is given by $\mathfrak{x} \mapsto \mathfrak{x} \otimes 1$.

\footnotetext{
${ }^{3}$ We use the right action here to match later with our convention for the action map of a crossed product, cf. 2.1.
} 
ON LOCALLY ANALYTIC BEILINSON-BERNSTEIN LOCALIZATION AND THE CANONICAL DIMENSION

Lemma 7.7. The action map $\sigma_{u}$ induces a group homomorphism

$$
H_{m} \rightarrow \operatorname{Out}\left(\widehat{\mathcal{U}_{n}^{\lambda}}\right) \text {. }
$$

The map $\tau_{u}$ is a 2-cocycle with respect to $\sigma_{u}$.

Proof. Let $\sigma^{\prime}: G \rightarrow \operatorname{Aut}\left(G^{p^{m}}\right)$ be the right action given by conjugation of $G$ on the normal subgroup $G^{p^{m}}$, i.e. $\sigma^{\prime}(g): h \mapsto g^{-1} h g$. It is easy to check that the identities

$$
\tau^{\prime}(x y, z) \cdot \tau^{\prime}(x, y)^{\sigma^{\prime}(z)}=\tau^{\prime}(x, y z) \cdot \tau^{\prime}(y, z), \quad \sigma^{\prime}(y) \sigma^{\prime}(z)=\sigma^{\prime}(y z) \eta(y, z)
$$

hold for all $x, y, z \in H_{m}$ where $\eta(y, z)$ denotes the automorphism induced by right conjugation with $\tau^{\prime}(y, z)$. It remains to observe that the homomorphism $\mathcal{L}_{G^{p}}^{-1}$ intertwines the conjugation action on the source with the adjoint action on the target.

According to the preceding lemma we have, for each $m \geq 1$, an associative crossed product

$$
A_{m}^{\lambda}:=\widehat{\mathcal{U}_{n}^{\lambda}} *_{\sigma_{u}, \tau_{u}} H_{m}
$$

where $n=(m-1) e+k \geq 1$ and $H_{m}=G / G^{p^{m}}$. We emphasize that the datum $\sigma_{u}, \tau_{u}$ depends on $m$. We put $A_{m, K}^{\lambda}:=A_{m}^{\lambda} \otimes_{o_{L}} K$ so that $A_{m, K}^{\lambda}=\widehat{\mathcal{U}_{n, K}^{\lambda}} * H_{m}$.

7.8. Recall the homomorphism

$$
\operatorname{res}_{u}: \widehat{\mathcal{U}_{n+1, K}^{\lambda}} \rightarrow \widehat{\mathcal{U}_{n, K}^{\lambda}}
$$

for all $n$ from the end of section 4. Using it we may define a $K$-linear homomorphism

$$
\text { res }_{a}: A_{m+1, K}^{\lambda} \longrightarrow A_{m, K}^{\lambda}
$$

compatible with res $_{u}$ as follows: by (7.5) the source is a finite free $\widehat{\mathcal{U}_{n+1}^{\lambda}}$-module on basis elements $\bar{g} \bar{h} \in \bar{H}_{m+1}$ where $\bar{g} \in \bar{H}_{m, m+1} \subset G^{p^{m}}$ and $\bar{h} \in \bar{H}_{m}$ and we put

$$
\operatorname{res}_{a}(\bar{g} \bar{h}):=L_{u}(\bar{g}) \cdot \bar{h}
$$

where $L_{u}$ equals the map

$$
G^{p^{m}} \stackrel{\mathcal{L}_{G^{m}}^{-1}}{\longrightarrow}{\widehat{U(\mathfrak{g})_{n}}}^{\times} \longrightarrow \widehat{\mathcal{U}}_{n}^{\times}
$$

used in the definition of $\tau_{u}$. It will follow from the proposition below that the $K$-linear map res $_{a}$ is actually an algebra homomorphism.

Consider the central character

$$
\theta: Z\left(\mathfrak{g}_{K}\right) \longrightarrow K
$$

that is induced from $\lambda_{K} \in \mathfrak{t}_{K}^{*}$ via the Harish-Chandrda map $\phi_{K}$ and recall the central reductions $D_{r_{m}}(G)_{\theta}$. Recall (5.13) the canonical inclusion $D_{r_{0}}\left(G^{p^{m}}\right) \hookrightarrow D_{r_{m}}(G)$. Cor. 6.13 and Prop. 7.3 imply the isomorphism

$$
\mathcal{L}_{G^{p}}: \widehat{\mathcal{U}_{n}^{\lambda}}, \stackrel{\cong}{\longrightarrow} D_{r_{0}}\left(G^{p^{m}}\right)_{\theta} .
$$


The two maps compose to an algebra homomorphism

$$
\widehat{\mathcal{U}_{n, K}^{\lambda}} \longrightarrow D_{r_{m}}(G)_{\theta} \text {. }
$$

Proposition 7.9. The homomorphism extends to an algebra isomorphism

$$
A_{m, K}^{\lambda} \stackrel{\cong}{\longrightarrow} D_{r_{m}}(G)_{\theta}
$$

which is compatible with variation in $m$. In particular, there is a canonical algebra isomorphism

$$
A_{K}^{\lambda}:={\underset{\lim }{m}}_{m, K} A_{m}^{\lambda} \stackrel{\cong}{\longrightarrow} D(G)_{\theta} .
$$

Proof. Source and target are crossed products involving the same group $H_{m}$. It can be checked that the corresponding actions and twistings are intertwined by the isomorphism $\mathcal{L}_{G^{p^{m}}}$. This yields the first assertion. The second assertion follows by inspection. The final assertion follows from (6.12) together with the fact that $r_{m} \uparrow 1$ for $m \rightarrow \infty$.

7.10. Recall that the sheaf $\tilde{\mathcal{D}}$ is (left) G-equivariant and, a fortiori, left $G$-equivariant. Since $G$ acts $o_{L}$-linearly this structure descends to the deformation $\tilde{\mathcal{D}}_{n}$ and then to the p-adic completion $\widehat{\widetilde{\mathcal{D}}}_{n}$. Since the image of

$$
\widehat{\psi_{n}}: \widehat{U(\mathfrak{t})_{n}} \longrightarrow \widehat{\tilde{\mathcal{D}}}_{n}
$$

lies in the $G$-invariants of $\widehat{\tilde{\mathcal{D}}}_{n}([38], \S 3)$ we obtain an equivariant structure on the central reduction

$$
\widehat{\mathcal{D}_{n}^{\lambda}}=\widehat{\tilde{\mathcal{D}}_{n}} \otimes_{\widehat{U(\mathfrak{t})_{n}, \lambda}} O_{K}
$$

by letting $G$ act on the left factor. Let $U \in \mathcal{S}$. The algebra $\widehat{\mathcal{D}_{n}^{\lambda}}(U)$ equals the $p$-adic completion of $\mathcal{D}_{n}^{\lambda}(U)$ where $\mathcal{D}_{n}^{\lambda}=\tilde{\mathcal{D}}_{n} \otimes_{U(\mathfrak{t})_{n}, \lambda} o_{K}$. Since $\mathcal{S}$ is a base for the topology on $X$, the sheaf $\widehat{\mathcal{D}_{n}^{\lambda}}$ is therefore supported only on the special fibre $X_{s}=X \times_{o_{L}} \kappa$ of the $o_{L}$-scheme $X$. However, the set $X_{s}$ is fixed pointwise by the group

$$
G\left(\pi_{L}\right)=\operatorname{ker}\left(\mathbf{G}\left(o_{L}\right) \longrightarrow \mathbf{G}(\kappa)\right) .
$$

Since $G \subseteq G\left(\pi_{L}\right)$ the equivariant $G$-structure is actually an action of $G$ on the sheaf $\widehat{\mathcal{D}_{n}^{\lambda}}$. Precomposing with the involution $g \mapsto g^{-1}$ we have a homomorphism

$$
\tilde{\sigma}: G \longrightarrow \operatorname{Aut}\left(\widehat{\mathcal{D}_{n}^{\lambda}}\right)
$$

(we remind the reader another time of our convention regarding the multiplication in automorphism groups, cf. section 2). Consequently, we have a map

$$
\sigma_{\mathcal{D}}: H_{m} \stackrel{s_{m}}{\longrightarrow} G \longrightarrow \operatorname{Aut}\left(\widehat{\mathcal{D}_{n}^{\lambda}}\right)
$$

where $s_{m}$ is our fixed section of the projection map $G \rightarrow H_{m}$. Recall that we have an algebra homomorphism

$$
\widehat{\varphi_{n}^{\lambda}}: \widehat{\mathcal{U}_{n}^{\lambda}} \longrightarrow \Gamma\left(X, \widehat{\mathcal{D}_{n}^{\lambda}}\right)
$$


which intertwines the (right) adjoint action on the source with our right action on the target (cf. [38], §3). We have a map

$$
\tau_{\mathcal{D}}: H_{m} \times H_{m} \stackrel{\tau_{u}}{\longrightarrow} \widehat{\mathcal{U}}_{n}^{\lambda} \stackrel{\widehat{\varphi_{n}^{\lambda}}}{\longrightarrow} \Gamma\left(X, \widehat{\mathcal{D}_{n}^{\lambda}}\right)^{\times}
$$

Lemma 7.11. The action map $\sigma_{\mathcal{D}}$ induces a group homomorphism

$$
H_{m} \rightarrow \operatorname{Out}\left(\widehat{\mathcal{D}_{n}^{\lambda}}\right)
$$

The map $\tau_{\mathcal{D}}$ is a 2-cocycle with respect to $\sigma_{\mathcal{D}}$.

Proof. Let $\sigma:=\sigma_{\mathcal{D}}, \tau:=\tau_{\mathcal{D}}$. We know that $\tau_{u}$ is a 2 -cocycle with respect to $\sigma_{u}$. By the intertwining property of $\widehat{\varphi_{n}^{\lambda}}$ mentioned above we obtain the first of the following two identities:

$$
\tau(x y, z) \cdot \tau(x, y)^{\sigma(z)}=\tau(x, y z) \cdot \tau(y, z), \quad \sigma(y) \sigma(z)=\sigma(y z) \eta(y, z),
$$

$x, y, z \in H_{m}$ where $\eta(y, z)$ denotes the automorphism of the sheaf $\widehat{\mathcal{D}_{n}^{\lambda}}$ induced by right conjugation with the unit $\tau(y, z)$. Let us show the second identity. By definition of $\tau^{\prime}$ we have in $G$

$$
\bar{y} \cdot \bar{z}=\overline{y z} \cdot \tau^{\prime}(y, z)
$$

Applying the homomorphism $\tilde{\sigma}$ we obtain

$$
\sigma(y) \sigma(z)=\sigma(y z) \tilde{\sigma}\left(\tau^{\prime}(y, z)\right) .
$$

We are therefore reduced to show

$$
\eta(y, z)=\tilde{\sigma}\left(\tau^{\prime}(y, z)\right)
$$

as automorphisms of the sheaf $\widehat{\mathcal{D}_{n}^{\lambda}}$. Since $\widehat{\mathcal{D}_{n}^{\lambda}}$ is supported on $X_{s}$, it suffices to prove this for the restriction of $\widehat{\mathcal{D}_{n}^{\lambda}}$ to $X_{s}([25]$, Prop. 6.4) which we denote by the same symbol. Choose an open subset $V \in \mathcal{S}$ and put $U:=V \cap X_{s}$. It suffices to check that both sides of $(*)$ induce the same automorphism of $\widehat{\mathcal{D}_{n}^{\lambda}}(U)$. This assertion is then seen most conceptually by introducing a certain completed skew group ring as follows (cf. [44], §3). To ease notation and to make the comparison with loc.cit. more transparent we will for the rest of this proof only consider left actions. Since $U$ is contained in the special fibre, the induced action of $G^{p^{m}}$ on $X$ fixes $U$ pointwise whence $G^{p^{m}}$ acts on $\mathcal{O}_{X}(U)$ by ring automorphisms. The derived action $\mathfrak{g} \rightarrow \mathcal{T}_{X}$ of $\mathbf{G}$ on $X$ induces, by restriction, an action of $\pi_{L}^{n} \mathfrak{g}$ on $\mathcal{O}_{X}(U)$ by $o_{L}$-derivations. We may therefore form the skew group ring and the skew enveloping algebra

$$
\mathcal{O}_{X}(U) \# G^{p^{m}}, \quad \mathcal{O}_{X}(U) \# U(\mathfrak{g})_{n} .
$$

with respect to these actions $([37], 1.5 .4,1.7 .10)$. Both rings have natural $G^{p^{m}}$-actions: given $g \in G^{p^{m}}$ let

$$
g \cdot(f \otimes h):=(g \cdot f) \otimes\left(g h g^{-1}\right)
$$


for $f \in \mathcal{O}_{X}(U), h \in G^{p^{m}}$ and

$$
g \cdot(f \otimes \mathfrak{x}):=(g \cdot f) \otimes(\operatorname{Ad}(g)(\mathfrak{x}))
$$

for $f \in \mathcal{O}_{X}(U), \mathfrak{x} \in U(\mathfrak{g})_{n}$. We let

$$
\iota: U(\mathfrak{g})_{n} \hookrightarrow \mathcal{O}_{X}(U) \# U(\mathfrak{g})_{n}, \mathfrak{x} \mapsto 1 \otimes \mathfrak{x} .
$$

Let $\widehat{\mathcal{O}_{X}(U)}$ be the $p$-adic completion of $\mathcal{O}_{X}(U)$. Denote the base change to $K$ of the $p$-adic completion of the skew enveloping algebra by $\widehat{\mathcal{O}_{X}(U)}{ }_{K} \# \widehat{U(\mathfrak{g})_{n}},{ }$. The $G^{p^{m}}$-action extends to this completion. There is a natural $G^{p^{m}}$-equivariant surjective algebra homomorphism

$$
p r: \widehat{\mathcal{O}_{X}(U)_{K}} \# \widehat{U(\mathfrak{g})_{n}, K} \longrightarrow \widehat{\tilde{\mathcal{D}}}_{n, K}(U)
$$

compatible with the maps $\iota$ and $\widehat{\varphi}_{n, K}$ (cf. (4.5) and [39], Lemma C.1.3).

The $G^{p^{m}}$-action on the Banach space $\left.\widehat{\mathcal{O}_{X}(U}\right)_{K}$ is induced from the rational G-action on $X$. It is therefore locally analytic as can be seen along the lines of (the proof of) [53], Prop. 2.1'. According to [44], 3.2 there is a natural structure of topological $K$-algebra on the completed tensor product

$$
\left.\widehat{\mathcal{O}_{X}(U)}\right)_{K} \# D_{r_{0}}\left(G^{p^{m}}\right):=\widehat{\mathcal{O}_{X}(U)}{ }_{K} \hat{\otimes}_{K} D_{r_{0}}\left(G^{p^{m}}\right)
$$

compatible with the two skew multiplication rings above via the maps

$$
G^{p^{m}} \hookrightarrow D_{r_{0}}\left(G^{p^{m}}\right)^{\times}, \quad \mathcal{L}_{G^{p^{m}}}: \widehat{U(\mathfrak{g})_{n}}, \stackrel{\cong}{\longrightarrow} D_{r_{0}}\left(G^{p^{m}}\right) .
$$

Note for the second compatibility that $\mathcal{L}_{G^{p}}$ is compatible with the inclusions $U(\mathfrak{g}) \subset$ $\operatorname{Lie}\left(G^{p^{m}}\right) \subset D\left(G^{p^{m}}\right)$ (prop. 6.3). The inclusion

$$
\left.\mathcal{O}_{X}(U)_{K} \# G^{p^{m}} \hookrightarrow \widehat{\mathcal{O}_{X}(U}\right)_{K} \# D_{r_{0}}\left(G^{p^{m}}\right)
$$

has dense image ([52], Lemma 3.1) and the $G^{p^{m}}$-action extends by continuity to the target. We summarize this discussion in the following commutative diagram

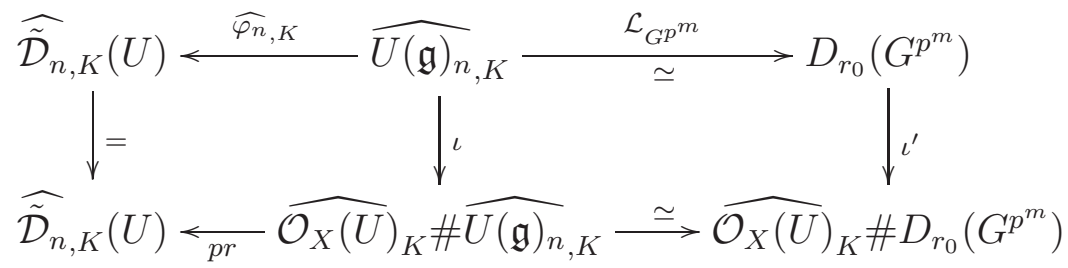

where $\iota^{\prime}(\delta):=1 \hat{\otimes} \delta$. The lower horizontal arrows are $G^{p^{m}}$-equivariant. Consider an element $g=\mathcal{L}_{G^{p}}(x) \in G^{p^{m}}$ and $\lambda \in \widehat{\mathcal{O}_{X}(U)_{K}} \# \widehat{U(\mathfrak{U})_{n}}$, . We claim

$$
g \cdot \lambda=\iota(x) \cdot \lambda \cdot \iota(x)^{-1} .
$$

According to the right-hand part of the above commutative diagram this can be checked in $\left.\widehat{\mathcal{O}_{X}(U}\right)_{K} \# D_{r_{0}}\left(G^{p^{m}}\right)$ where the identity reads

$$
g \cdot \lambda=\iota^{\prime}(g) \cdot \lambda \cdot \iota^{\prime}(g)^{-1} .
$$


By a density argument using again [52, Lemma 3.1 it suffices to check this identity on elements of the form $\lambda=f \otimes \delta_{h}$ with $f \in \mathcal{O}_{X}(U), \delta_{h} \in G^{p^{m}}$, i.e. in the skew group ring $\mathcal{O}_{X}(U) \# G^{p^{m}}$. Denoting by $\cdot$ the skew multiplication in the latter ring we have

$$
g \cdot\left(f \cdot \delta_{h}\right)=(g \cdot f) \cdot\left(g \delta_{h} g^{-1}\right)=g \cdot f \cdot g^{-1} \cdot\left(g \delta_{h} g^{-1}\right)=g \cdot\left(f \cdot \delta_{h}\right) \cdot g^{-1}
$$

which is the assertion. Using the left-hand part of the above commutative diagram we deduce that a group element $g=\mathcal{L}_{G^{p}}(x) \in G^{p^{m}}$ acts on $\widehat{\tilde{\mathcal{D}}}_{n, K}(U)$ by conjugation with $\widehat{\varphi_{n}, K}(x)$. Passing to central reductions via $\lambda$ we see that such an element acts on $\widehat{\mathcal{D}_{n, K}^{\lambda}}(U)$ by conjugation with $\widehat{\varphi_{n, K}^{\lambda}}(x)$. Applying this to the element $\tau_{u}(y, z) \in \widehat{\mathcal{U}_{n}^{\lambda}, K}$ and observing that $\mathcal{L}_{G^{p}} \circ \tau_{u}=\tau^{\prime}$ (cf. (77.6) ) we see that $\tau^{\prime}(y, z)$ acts from the left on $\widehat{\tilde{\mathcal{D}}}_{n, K}(U)$ by left conjugation with the unit $\widehat{\varphi_{n, K}^{\lambda}}\left(\tau_{u}(y, z)\right)=\tau(y, z)$ which is what we want.

To ease notation we abbreviate $\sigma:=\sigma_{\mathcal{D}}, \tau:=\tau_{\mathcal{D}}$ in the following. By section 2 and the lemma we have, for each $m \geq 1$, a crossed product sheaf of associative $K$-algebras

$$
\mathcal{A}_{m, K}^{\lambda}:=\widehat{\mathcal{D}_{n, K}^{\lambda}} *_{\sigma, \tau} H_{m}
$$

where $n=(m-1) e+k$ and $H_{m}=G / G^{p^{m}}$. We emphasize that the datum $\sigma, \tau$ depends on $m$. Since $\widehat{\mathcal{D}_{n, K}^{\lambda}}$ is a sheaf of coherent rings, so is $\mathcal{A}_{m, K}^{\lambda}$.

7.12. Let us assume that the weight $\lambda+\rho \in \mathfrak{t}_{K}^{*}$ is dominant and regular. Since $\mathcal{A}_{m, K}^{\lambda}$ is a finite free (left) module over $\widehat{\mathcal{D}_{n, K}^{\lambda}}$, the equivalence of categories for $\widehat{\mathcal{D}_{n, K}^{\lambda}}$ given in Thm. 4.9 admits the following straightforward version over the sheaf of rings $\mathcal{A}_{m, K}^{\lambda}$. Recall the functor $\operatorname{Loc}_{\lambda}$ of (4.11). Given a coherent $\Gamma\left(X, \mathcal{A}_{m, K}^{\lambda}\right)$-module $M$ we may form the $\mathcal{A}_{m, K^{-}}^{\lambda}$ module

$$
\mathrm{L}_{\lambda}(M):=\mathcal{A}_{m, K}^{\lambda} \otimes_{\Gamma\left(\mathcal{A}_{m, K}^{\lambda}\right)} M
$$

Any element $\mu$ in this module can be written as

$$
\sum_{k, i} \lambda_{k}(\mu) \bar{h}_{k} \otimes m_{i}=\sum_{k, i} \lambda_{k}(\mu) \otimes \bar{h}_{k} \cdot m_{i}
$$

with $\lambda(\mu)_{k} \in \widehat{\mathcal{U}_{n, K}^{\lambda}}, m_{i} \in M$. The natural homomorphism

$$
\operatorname{Loc}_{\lambda}(M) \rightarrow \mathrm{L}_{\lambda}(M)
$$

of $\widehat{\mathcal{D}_{n, K}^{\lambda}}$-modules induced by the embedding $\widehat{\mathcal{D}_{n, K}^{\lambda}} \hookrightarrow \mathcal{A}_{m, K}^{\lambda}$ is therefore surjective. Since $\operatorname{Loc}_{\lambda}(M)$ is a coherent $\widehat{\mathcal{D}_{n, K}^{\lambda}}$-module (Thm. [4.9), $\mathrm{L}_{\lambda}(M)$ is a coherent $\mathcal{A}_{m, K^{\lambda}}^{\lambda}$ module. In a similar manner we deduce that $\Gamma(\mathcal{M})$ is a coherent $\Gamma\left(\mathcal{A}_{m, K}^{\lambda}\right)$-module for any coherent $\mathcal{A}_{m, K^{\lambda}}^{\lambda}$-module $\mathcal{M}$. We therefore have the adjoint pair $\left(\mathrm{L}_{\lambda}, \Gamma(\cdot)\right)$ between the abelian categories $\operatorname{coh}\left(\Gamma\left(\mathcal{A}_{m, K}^{\lambda}\right)\right)$ and $\operatorname{coh}\left(\mathcal{A}_{m, K}^{\lambda}\right)$. 
Proposition 7.13. Suppose the weight $\lambda+\rho \in \mathfrak{t}_{K}^{*}$ is dominant and regular. The pair $\left(\mathrm{L}_{\lambda}, \Gamma(\cdot)\right)$ induces mutually inverse equivalence of categories

$$
\operatorname{coh}\left(\Gamma\left(\mathcal{A}_{m, K}^{\lambda}\right)\right) \stackrel{\cong}{\longrightarrow} \operatorname{coh}\left(\mathcal{A}_{m, K}^{\lambda}\right) .
$$

Proof. By Thm. 4.9 the functor $\Gamma(\cdot)$ is exact and has no kernel. By [6], Lemma 2.4 it suffices to check that the counit $\eta_{M}: M \rightarrow \Gamma\left(\mathrm{L}_{\lambda}(M)\right)$ is an isomorphism for $M \in$ $\operatorname{coh}\left(\Gamma\left(\mathcal{A}_{m, K}^{\lambda}\right)\right)$. Since $M$ is coherent, it has a finite free presentation as $\Gamma\left(\mathcal{A}_{m, K}^{\lambda}\right)$-module. Since $\eta_{\Gamma\left(\mathcal{A}_{m, K}^{\lambda}\right)}$ is obviously an isomorphism and $\Gamma\left(\mathrm{L}_{\lambda}(\cdot)\right)$ is right-exact, the claim follows from the Five Lemma.

7.14. Let $U \in \mathcal{S}$ and $h \in H_{m}$. Since $\sigma(h)$ preserves the unit ball $\widehat{\mathcal{D}_{n}^{\lambda}}(U)$ of the $K$-Banach algebra $\widehat{\mathcal{D}_{n, K}^{\lambda}}(U)$ it acts on the latter by isometries. Recall (cf. (4.14)) the algebra homomorphism

$$
r e s_{\mathcal{D}}: \widehat{\mathcal{D}_{n+1, K}^{\lambda}} \longrightarrow \widehat{\mathcal{D}}_{n, K}^{\lambda}
$$

induced by $\tilde{\mathcal{D}}_{n+1} \subseteq \tilde{\mathcal{D}}_{n}$. Using this homomorphism we may define a $K$-linear homomorphism

$$
\operatorname{res}_{\mathcal{A}}: \mathcal{A}_{m+1, K}^{\lambda} \longrightarrow \mathcal{A}_{m, K}^{\lambda}
$$

compatible with $\operatorname{res}_{\mathcal{D}}$ by a procedure very similar to the one we used for the rings $A_{n, K}^{\lambda}$. Indeed, the source is a finite free $\widehat{\mathcal{D}_{n+1, K}^{\lambda}}$-module on basis elements $\bar{g} \bar{h} \in \bar{H}_{m+1}$ where $\bar{g} \in \bar{H}_{m, m+1} \subset G^{p^{m}}$ and $\bar{h} \in \bar{H}_{m}$ and we put

$$
\operatorname{res}_{\mathcal{A}}(\bar{g} \bar{h}):=L_{\mathcal{D}}(\bar{g}) \cdot \bar{h}
$$

where $L_{\mathcal{D}}$ equals the composite of the map

$$
L_{u}: G^{p^{m}} \stackrel{\mathcal{L}_{G^{m}}^{-1}}{\longrightarrow}{\widehat{U(\mathfrak{g})_{n}}}^{x} \longrightarrow \widehat{\mathcal{U}}_{n}^{\lambda} \times
$$

used in the definition of $\tau_{u}$ and the map ${\widehat{\varphi_{n}^{\lambda}}}: \widehat{\mathcal{U}}_{n}^{\lambda} \rightarrow \Gamma\left(X, \widehat{\mathcal{D}_{n}^{\lambda}}\right)^{\times}$.

Lemma 7.15. The morphism res $_{\mathcal{A}}$ is multiplicative.

Proof. Let $\bar{g} \in \bar{H}_{m, m+1} \subset G^{p^{m}}, \bar{h} \in \bar{H}_{m}$ and $\lambda$ a local section of $\widehat{\mathcal{D}_{n+1, K}^{\lambda}}$. By definition

$$
\operatorname{res}_{\mathcal{A}}(\bar{h} \cdot \sigma(h)(\lambda))=\operatorname{res}_{\mathcal{A}}(\lambda \cdot \bar{h})=\operatorname{res}_{\mathcal{D}}(\lambda) \cdot \bar{h}
$$

and the right-hand side equals, by equivariance of $r e s_{\mathcal{D}}$,

$$
\bar{h} \cdot \sigma(h)\left(\operatorname{res}_{\mathcal{D}}(\lambda)\right)=\operatorname{res}_{\mathcal{A}}(\bar{h}) \cdot \operatorname{res}_{\mathcal{A}}(\sigma(h)(\lambda)) .
$$

Similarly,

$$
\operatorname{res}_{\mathcal{A}}(\bar{g} \cdot \sigma(g)(\lambda))=\operatorname{res}_{\mathcal{A}}(\lambda \cdot \bar{g})=\operatorname{res}_{\mathcal{D}}(\lambda) \cdot L_{\mathcal{D}}(\bar{g})
$$


We have shown in the proof of lemma 7.11 that the group element $g$ operates on the local section $\operatorname{res}_{\mathcal{D}}(\lambda)$ with respect to the $G$-structure on the sheaf $\widehat{\mathcal{D}_{n, K}^{\lambda}}$ through conjugation by the global unit $L_{\mathcal{D}}(\bar{g}) \in \Gamma\left(X, \widehat{\mathcal{D}_{n, K}^{\lambda}}\right)^{\times}$. This implies

$$
\operatorname{res}_{\mathcal{D}}(\lambda) \cdot L_{\mathcal{D}}(\bar{g})=L_{\mathcal{D}}(\bar{g}) \cdot \sigma(g)\left(\operatorname{res}_{\mathcal{D}}(\lambda)\right)=\operatorname{res}_{\mathcal{A}}(\bar{g}) \cdot \operatorname{res}_{\mathcal{A}}(\sigma(g)(\lambda)) .
$$

Finally, we have to show that res $\mathcal{A}$ respects products of the form $\bar{h}_{1} \cdot \bar{h}_{2}$ with $h_{1}, h_{2} \in \bar{H}_{m+1}$. Since the map res $s_{a}$ from 7.8 is multiplicative we have $\operatorname{res}_{a}\left(\bar{h}_{1}\right) \cdot \operatorname{res}_{a}\left(\bar{h}_{2}\right)=\operatorname{res}_{a}\left(\overline{h_{1}} \cdot \overline{h_{2}}\right)$. Applying the multiplicative map $\widehat{\varphi_{n, K}^{\lambda}}$ and the preceding lemma yields the claim.

We therefore have the projective system $\left(\mathcal{A}_{m, K}^{\lambda}\right)_{m}$ of sheaves of $K$-algebras. By Cor. 4.16 and Prop. 2.12 it satisfies the conditions (i),(ii),(iii) of section 2. Hence, letting

$$
\mathcal{A}_{K}^{\lambda}:={\underset{m}{m}}_{\lim _{m, K}} \mathcal{A}_{m, \lambda}^{\lambda}
$$

be the projective limit we have the chain of full abelian subcategories

$$
\operatorname{coh}\left(\mathcal{A}_{K}^{\lambda}\right) \subset \mathscr{C}_{\mathcal{A}_{K}^{\lambda}} \subset \operatorname{Mod}\left(\mathcal{A}_{K}^{\lambda}\right)
$$

and the equivalence of categories

$$
\Gamma: \operatorname{coh}\left(\left(\mathcal{A}_{m, K}^{\lambda}\right)_{m}\right) \stackrel{\cong}{\longrightarrow} \mathscr{C}_{\mathcal{A}_{K}^{\lambda}} .
$$

7.17. We shall need a simple lemma on the behaviour of the categories $\operatorname{coh}\left(\mathcal{A}_{m, K}^{\lambda}\right)$ relative to the restriction maps res $\mathcal{A}$. Let $\left(\mathcal{M}_{m}\right)_{m}$ be a projective system with $\mathcal{M}_{m} \in \operatorname{coh}\left(\mathcal{A}_{m, K}^{\lambda}\right)$. We have a natural map of $\Gamma\left(\mathcal{A}_{m, K}^{\lambda}\right)$-modules

$$
\Gamma\left(\mathcal{A}_{m, K}^{\lambda}\right) \otimes_{\Gamma\left(\mathcal{A}_{m+1, K}^{\lambda}\right)} \Gamma\left(\mathcal{M}_{m+1}\right) \longrightarrow \Gamma\left(\mathcal{A}_{m, K}^{\lambda} \otimes_{\mathcal{A}_{m+1, K}^{\lambda}} \mathcal{M}_{m+1}\right) .
$$

Lemma 7.18. Suppose the weight $\lambda+\rho \in \mathfrak{t}_{K}^{*}$ is dominant and regular. The above map is an isomorphism.

Proof. The sheaf $\mathcal{A}_{m, K}^{\lambda}$ is a sheaf of coherent rings. The $\mathcal{A}_{m, K}^{\lambda}$-module $\mathcal{A}_{m, K}^{\lambda} \otimes_{\mathcal{A}_{m+1, K}^{\lambda}} \mathcal{M}_{m+1}$ is hence of finite presentation and therefore coherent (cf. (2.4)). According to the above proposition, $\Gamma(\cdot)$ is exact on coherent modules over either $\mathcal{A}_{m, K}^{\lambda}$ or $\mathcal{A}_{m+1, K}^{\lambda}$ and therefore both sides of our map are right-exact functors in $\mathcal{M}_{m+1}$. By the above proposition, we have a global finite presentation $\left(\mathcal{A}_{m+1, K}^{\lambda}\right)^{b} \rightarrow\left(\mathcal{A}_{m+1, K}^{\lambda}\right)^{a} \rightarrow \mathcal{M}_{m+1} \rightarrow 0$. The Five Lemma reduces us therefore to the case $\mathcal{M}_{m+1}=\mathcal{A}_{m+1, K}^{\lambda}$ which is obvious.

7.19. We now compute the global sections of the sheaf $\mathcal{A}_{K}^{\lambda}$ under the hypothesis (H1)(H3) of (4.10). To this end, recall the projective system

$$
A_{m, K}^{\lambda}=\widehat{\mathcal{U}_{n, K}^{\lambda}} *_{\sigma_{u}, \tau_{u}} H_{m}
$$

where $n=(m-1) e+k$ and $H_{m}=G / G^{p^{m}}$. The algebra homomorphism

$$
\widehat{\varphi_{n, K}^{\lambda}}: \widehat{\mathcal{U}_{n, K}^{\lambda}} \longrightarrow \widehat{\mathcal{D}}_{n, K}^{\lambda}
$$


extends to a linear map

$$
A_{m, K}^{\lambda} \longrightarrow \mathcal{A}_{m, K}^{\lambda}
$$

preserving the basis elements $\bar{h}$ for $h \in H_{m}$ on both sides.

Lemma 7.20. The linear map $(*)$ is multiplicative and compatible with variation in $m$.

Proof. The map $(*)$ respects the action maps $\sigma_{u}, \sigma$ since $\widehat{\varphi_{n, K}^{\lambda}}$ intertwines the adjoint action with the $G$-action on the target. Since moreover $\tau=\widehat{\varphi_{n}^{\lambda}} \circ \tau_{u}$ the map (*) is multiplicative. The final assertion is obvious from $L_{\mathcal{D}}=\widehat{\varphi_{n}^{\lambda}} \circ L_{u}$.

Corollary 7.21. If (H1)-(H3) hold, then (*) induces a algebra isomorphisms

$$
A_{m, K}^{\lambda} \stackrel{\cong}{\longrightarrow} \Gamma\left(X, \mathcal{A}_{m, K}^{\lambda}\right)
$$

for all $m$ and, in the projective limit, an algebra isomorphism

$$
D(G)_{\theta} \stackrel{\cong}{\longrightarrow} \Gamma\left(X, \mathcal{A}_{K}^{\lambda}\right) .
$$

Proof. The first statement follows from Thm. 4.11. The second statement follows then from the preceding lemma and Prop. 7.9.

7.22. Let now $G=G\left(\pi_{L}^{k}\right)$ be an any congruence subgroup for $k \geq 1$. The above discussion for the case $k \geq e$ extends in an obvious way to the general case as follows. Let $\tilde{G}=G\left(\pi_{L}^{\tilde{k}}\right)$ for $\tilde{k} \geq \max (e, k)$ and let $H_{m}, A_{m, K}^{\lambda}, A_{K}^{\lambda}, \mathcal{A}_{m, K}^{\lambda}, \mathcal{A}_{K}^{\lambda}$ be formed relative to $\tilde{G}$ as described above. We have the finite group $G_{m}=G / \tilde{G}^{p^{m}}$ containing $H_{m}$ as a normal subgroup. We choose a system of representatives $\mathcal{R}$ for $G_{m} / H_{m}$ in $G$ containing $1 \in G$ and let $\bar{G}_{m}=\bar{H}_{m} \cdot \mathcal{R}$ (in obvious notation) be the induced system of representatives of $G_{m}$ in $G$. This extends the maps $s_{m}$ and $\tau^{\prime}$ from $H_{m}$ to $G_{m}$. We therefore obtain extensions of $\sigma_{u}$ and $\tau_{u}$ to $G_{m}$ and corresponding crossed products $B_{m, K}^{\lambda}=\widehat{\mathcal{U}_{n, K}^{\lambda}} * G_{m}$ and $\mathcal{B}_{m, K}^{\lambda}=\widehat{\mathcal{D}_{n, K}^{\lambda}} * G_{m}$. According to [43], Lemma 1.3 these are actually crossed products over $A_{m, K}^{\lambda}$ and $\mathcal{A}_{m, K}^{\lambda}$ relative to the finite group $G_{m} / H_{m}=G / \tilde{G}$ respectively. Passing to the limit over $m$ we obtain crossed products

$$
B_{K}^{\lambda}=A_{K}^{\lambda} *(G / \tilde{G}) \text { and } \mathcal{B}_{K}^{\lambda}=\mathcal{A}_{K}^{\lambda} *(G / \tilde{G}) .
$$

Using $D(G)_{\theta}=D(\tilde{G})_{\theta} *(G / \tilde{G})$ one deduces, in case $(H 1)-(H 3)$ hold, without difficulty an algebra isomorphism $D(G)_{\theta} \cong \Gamma\left(X, \mathcal{B}_{K}^{\lambda}\right)$ extending Cor. 7.21. Finally, if $\lambda+\rho \in \mathfrak{t}_{K}^{*}$ is dominant and regular, we have the analogues of Prop. 7.13 and Lem. 7.18 for the projective system $\left(\mathcal{B}_{m, K}^{\lambda}\right)_{m}$ which follow with the same proofs.

\section{The Equivalence of CATEgories}

Let in the following

$$
G=G\left(\pi_{L}^{k}\right)=\operatorname{ker}\left(\mathbf{G}\left(o_{L}\right) \longrightarrow \mathbf{G}\left(o_{L} / \pi_{L}^{k} o_{L}\right)\right)
$$


be a congruence subgroup for some $k \geq 1$. We assume $p \neq 2$ and that the hypothesis (H1)-(H3) of (4.10) are satisfied.

8.1. Recall that $Z\left(\mathfrak{g}_{K}\right)$ equals the center of the universal enveloping algebra $U\left(\mathfrak{g}_{K}\right)$ and that $Z\left(\mathfrak{g}_{K}\right)$ lies in the center of the ring $D(G)$ ([52], Prop. 3.7). We fix a character

$$
\theta: Z\left(\mathfrak{g}_{K}\right) \longrightarrow K
$$

and the central reduction

$$
D(G)_{\theta}:=D(G) \otimes_{Z\left(\mathfrak{g}_{K}\right), \theta} K
$$

of $D(G)$. As we have explained the algebra $D(G)_{\theta}=\lim _{m} D_{r_{m}}(G)_{\theta}$ is again Fréchet-Stein and its coadmissible modules

$$
\mathscr{C}_{G, \theta}=\mathscr{C}_{G} \cap \operatorname{Mod}\left(D(G)_{\theta}\right)
$$

are in duality with admissible locally analytic $G$-representations having infinitesimal character $\theta$.

Remark: Building on a p-adic version due to Ardakov-Wadsley ([4], §8) of the famous Quillen's lemma it is shown in the preprint [19] that any topologically irreducible admissible locally analytic $G$-representation admits, up to a finite extension of $K$, an infinitesimal character.

Up to a finite extension of $K$ there is an element $\lambda \in \mathfrak{t}_{K}^{*}$ that pulls back to $\theta$ under the Harish-Chandra homomorphism $\phi_{K}$. We fix such a $\lambda$. There is then a minimal $m(\lambda) \geq 0$ such that $\lambda\left(\pi_{L}^{m(\lambda)} \mathfrak{t}\right) \subseteq o_{K}$, i.e.

$$
\lambda \in \operatorname{Hom}_{o_{L}}\left(\pi_{L}^{m} \mathfrak{t}, o_{K}\right)
$$

for all $m>m(\lambda)$. We tacitly restrict in the following to numbers $m>m(\lambda)$. According to the final paragraph in the preceding section, we may associate to $G$ a sheaf of rings $\mathcal{A}_{K}^{\lambda}$ on $X$ which comes with an isomorphism

$$
D(G)_{\theta} \stackrel{\cong}{\longrightarrow} \Gamma\left(X, \mathcal{A}_{K}^{\lambda}\right)
$$

and an abelian category of coadmissible sheaves $\mathscr{C}_{\mathcal{A}_{K}^{\lambda}}$. It allows to introduce the functor

$$
\mathrm{L}_{\lambda}: \operatorname{Mod}\left(D(G)_{\theta}\right) \rightarrow \operatorname{Mod}\left(\mathcal{A}_{K}^{\lambda}\right), \quad M \mapsto \mathcal{A}_{K}^{\lambda} \otimes_{D(G)_{\theta}} M
$$

It is left adjoint to the global section functor $\Gamma(X,$.$) and therefore right exact. The two$ units of the adjunction induce natural maps

$$
M \longrightarrow \Gamma \circ \mathrm{L}_{\lambda}(M), \quad \mathrm{L}_{\lambda} \circ \Gamma(\mathcal{M}) \longrightarrow \mathcal{M}
$$

for any $M \in \operatorname{Mod}\left(D(G)_{\theta}\right)$ and $\mathcal{M} \in \operatorname{Mod}\left(\mathcal{A}_{K}^{\lambda}\right)$. We do not know whether $\mathrm{L}_{\lambda}(M) \in \mathscr{C}_{\mathcal{A}_{K}^{\lambda}}$ for a general $M \in \mathscr{C}_{G, \theta}$. However, we still have following.

Theorem 8.2. Suppose the weight $\lambda+\rho \in \mathfrak{t}_{K}^{*}$ is dominant and regular. The functor $\Gamma(X,$. induces an equivalence of categories

$$
\mathscr{C}_{\mathcal{A}_{K}^{\lambda}} \stackrel{\cong}{\longrightarrow} \mathscr{C}_{G, \theta} .
$$


A quasi-inverse is given by the functor

$$
\left.\tilde{\mathrm{L}}_{\lambda}: M \mapsto{\underset{\mathrm{lim}}{m}}_{\lim _{m, K}^{\lambda}} \otimes_{D(G)_{\theta}} M\right) .
$$

If $M$ is a finitely presented $D(G)_{\theta}$-module the natural morphism $\mathrm{L}_{\lambda}(M) \stackrel{\cong}{\longrightarrow} \tilde{\mathrm{L}}_{\lambda}(M)$ is an isomorphism.

Proof. Let $\left(\mathcal{M}_{m}\right)_{m}$ be a projective system with coherent $\mathcal{A}_{m, K^{-}}^{\lambda}$ modules $\mathcal{M}_{m}$. Put $M_{m}:=$ $\Gamma\left(\mathcal{M}_{m}\right) \in \operatorname{Mod}^{\mathrm{fg}}\left(A_{m, K}^{\lambda}\right)$. According to the Cor. 7.18 the natural map

$$
A_{m, K}^{\lambda} \otimes_{A_{m+1, K}^{\lambda}} M_{m+1} \longrightarrow M_{m}
$$

is an isomorphism precisely when the natural map

$$
\mathcal{A}_{m, K}^{\lambda} \otimes_{\mathcal{A}_{m+1, K}^{\lambda}} \mathcal{M}_{m+1} \longrightarrow \mathcal{M}_{m}
$$

is an isomorphism, i.e. precisely when $\left(\mathcal{M}_{m}\right)_{m} \in \operatorname{coh}\left(\left(\mathcal{A}_{m, K}^{\lambda}\right)_{m}\right)$. This means that the collection of category equivalences $\operatorname{coh}\left(\mathcal{A}_{m, K}^{\lambda}\right) \stackrel{\cong}{\longrightarrow} \operatorname{Mod}^{\mathrm{fg}}\left(A_{m, K}^{\lambda}\right)$ induced by $\Gamma(\cdot)$ induces a category equivalence

$$
\left(\mathcal{M}_{m}\right)_{m} \mapsto\left(\Gamma\left(\mathcal{M}_{m}\right)\right)_{m}
$$

between $\operatorname{coh}\left(\left(\mathcal{A}_{m, K}^{\lambda}\right)_{m}\right)$ and the abelian category of families $\left(M_{m}\right)_{m}$ of finitely generated $A_{m, K}^{\lambda}$-modules with the property $A_{m, K}^{\lambda} \otimes_{A_{m+1, K}^{\lambda}} M_{m+1} \stackrel{\cong}{\longrightarrow} M_{m}$. By definition the latter equals the category of coherent sheafs for the distribution algebra $D(G)_{\theta}$. We therefore have the equivalence

given by the composite

$$
\mathscr{C}_{\mathcal{A}_{K}^{\lambda}} \stackrel{\cong}{\longrightarrow} \mathscr{C}_{G, \theta}
$$

$$
\mathcal{M} \mapsto\left(\mathcal{A}_{m, K}^{\lambda} \otimes_{\mathcal{A}_{K}^{\lambda}} \mathcal{M}\right)_{m} \mapsto\left(\Gamma\left(\mathcal{A}_{m, K}^{\lambda} \otimes_{\mathcal{A}_{K}^{\lambda}} \mathcal{M}\right)\right)_{m} \mapsto \underset{m}{\lim } \Gamma\left(\mathcal{A}_{m, K}^{\lambda} \otimes_{\mathcal{A}_{K}^{\lambda}} \mathcal{M}\right)
$$

and the right-hand term equals $\Gamma\left(\lim _{m} \mathcal{A}_{m, K}^{\lambda} \otimes_{\mathcal{A}_{K}^{\lambda}} \mathcal{M}\right)=\Gamma(\mathcal{M})$ according to Prop. 2.8. From the definitions we read off that a quasi-inverse is given by the functor

$$
\tilde{\mathrm{L}}_{\lambda}: M \mapsto \underset{m}{\lim _{m}}\left(\mathcal{A}_{m, K}^{\lambda} \otimes_{D(G)_{\theta}} M\right) \text {. }
$$

We have a natural morphism $f: \mathrm{L}_{\lambda}(M) \rightarrow \tilde{\mathrm{L}}_{\lambda}(M)$ induced by the maps $\mathcal{A}_{K}^{\lambda} \rightarrow \mathcal{A}_{m, K}^{\lambda}$ where the target is coadmissible. If $M$ is finitely presented, the source is also coadmissible. Since the morphism becomes an isomorphism for all $m$ after applying $\mathcal{A}_{m, K}^{\lambda} \otimes_{\mathcal{A}_{K}^{\lambda}}(\cdot)$, it is an isomorphism.

Corollary 8.3. Let $\operatorname{Rep}(G)_{\theta}$ be the category of admissible locally analytic G-representations over $K$ with infinitesimal character $\theta$. Let $\lambda \in \mathfrak{t}_{K}^{*}$ be a weight that corresponds to $\theta$ under the (untwisted) Harish-Chandra homomorphism. Suppose $\lambda+\rho$ is dominant and regular. There is an equivalence of categories

$$
\mathscr{C}_{\mathcal{A}_{K}^{\lambda}} \stackrel{\cong}{\longrightarrow} \operatorname{Rep}(G)_{\theta}
$$


given by $\mathcal{M} \mapsto \Gamma(X, \mathcal{M})_{b}^{\prime}$.

8.4. The completion $\hat{U}\left(\mathfrak{g}_{K}\right)$ of $U\left(\mathfrak{g}_{K}\right)$ with respect to all submultiplicative seminorms is called the Arens-Michael envelope of the $p$-adic Lie algebra $\mathfrak{g}_{K}$ and was studied in the papers [45], 48]. It is a Fréchet-Stein algebra and we have the abelian category $\mathscr{C}\left(\hat{U}\left(\mathfrak{g}_{K}\right)_{\theta}\right)$ of coadmissible modules over the central reduction $\hat{U}\left(\mathfrak{g}_{K}\right)_{\theta}$ at the infinitesimal character $\theta$. Arguing as in [45], Prop. 3.2.3 and using the formula $(*)$ in the proof of [54], Prop. 3.7 we see that $\hat{U}\left(\mathfrak{g}_{K}\right)_{\theta}$ is canonically isomorphic to the projective limit of the $\widehat{\mathcal{U}_{n}^{\lambda}, K}$. On the other hand, we may form the projective limit $\widehat{\mathcal{D}_{K}^{\lambda}}$ of the $\widehat{\mathcal{D}_{n, K}^{\lambda}}$. According to Cor. 4.16 we have the chain of full abelian subcategories

$$
\operatorname{coh}\left(\widehat{\mathcal{D}_{K}^{\lambda}}\right) \subset \mathscr{C}_{\widehat{\mathcal{D}_{K}^{\lambda}}} \subset \operatorname{Mod}\left(\widehat{\mathcal{D}_{K}^{\lambda}}\right)
$$

The commutative diagram at the end of section 4 induces a map $\hat{U}\left(\mathfrak{g}_{K}\right)_{\theta} \rightarrow \widehat{\mathcal{D}_{K}^{\lambda}}$. According to Thm. 4.9, it is an isomorphism on global sections in case (H1)-(H3) hold. Completely similar to the above discussion one may prove in this case that, if $\lambda+\rho$ is dominant and regular, then the functor $M \mapsto \lim _{n}\left(\widehat{\mathcal{U}_{n, K}^{\lambda}} \otimes_{\hat{U}\left(\mathfrak{g}_{K}\right)_{\theta}} M\right)$ induces an equivalence of categories

$$
\mathscr{C}\left(\hat{U}\left(\mathfrak{g}_{K}\right)_{\theta}\right) \stackrel{\cong}{\longrightarrow} \mathscr{C} \widehat{\mathcal{D}_{K}^{\lambda}}
$$

with quasi-inverse given by $\Gamma(X,$.$) . This equivalence is compatible with Thm. 8.2$ in the obvious way.

\section{Dimension COMputations}

As an application of the above methods we prove in this final section a locally analytic version of Smith's theorem ([56]). First, we have to recall some notation and properties concerning the canonical dimension. The canonical dimension (or rather the codimension) in the context of locally analytic representations appears first in [54], $\S 8$.

9.1. We recall (cf. [35], chap. III.) the notion of an Auslander regular ring. Let $R$ be an arbitrary associative unital ring. For any (left or right) $R$-module $N$ the grade $j_{R}(N)$ is defined to be either the smallest integer $l$ such that $\operatorname{Ext}_{R}^{l}(N, R) \neq 0$ or $\infty$. A left and right noetherian ring $R$ is called left and right Auslander regular if its left and right global dimension is finite and if every finitely generated left or right $R$-module $N$ satisfies Auslander's condition: for any $l \geq 0$ and any $R$-submodule $L \subseteq \operatorname{Ext}_{R}^{l}(N, R)$ one has $j_{R}(L) \geq l$.

In the following the term module always means left module. Noetherian rings are twosided noetherian and other ring-theoretic properties such as Auslander regular are used similarly.

Let $R$ be an Auslander regular ring and $M$ a finitely generated $R$-module. The number

$$
d_{R}(M):=\operatorname{gld}(R)-j_{R}(M)
$$


is called the canonical dimension of $M$. The map $M \mapsto d_{R}(M)$ is a finitely partitive exact dimension function in the sense of [37], $\$ 6.8 .4$ and $\S 8.3 .17$. For this and more details on the canonical dimension for finitely generated modules over an Auslander regular ring (or more generally, an Auslander-Gorenstein ring) we refer to [34].

A commutative noetherian ring is Auslander regular if and only if it is regular ([35], III.2.4.3). Let $R$ be an associative ring endowed with a separated exhaustive $\mathbb{Z}$-filtration by additive subgroups such that $R$ is complete in the filtration topology. If the associated graded ring of $R$ is Auslander regular of (left and right) global dimension $d$ then $R$ is Auslander regular and has (left and right) global dimension $\leq d$ ([loc.cit.], II.2.2.1, II.3.1.4, III.2.2.5).

9.2. Let now $G$ be a locally $L$-analytic group which is $L$-uniform. Recall that the rings underlying the $K$-Banach algebras $D_{r_{0}}\left(G^{p^{m}}\right)$ and $D_{r_{m}}(G)$ are noetherian ([54], Thm. 4.5).

Proposition 9.3. Let $m \geq 1$ and let $A$ denote one of the rings $D_{r_{0}}\left(G^{p^{m}}\right)$ or $D_{r_{m}}(G)$. Then $A$ is Auslander regular of (left and right) global dimension equal to $d=\operatorname{dim}_{L} G$. A finitely generated $A$-module $M$ is finite dimensional over $K$ if and only if $d_{A}(M)=0$.

Proof. By Lem. 5.11 the ring $D_{r_{m}}(G)$ is a finite free extension of $D_{r_{0}}\left(G^{p^{m}}\right)$ on a basis a system of representatives for the group $G / G^{p^{m}}$. By [54], Lem. 8.8 and [46], Cor. 7.3 it therefore suffices to prove the proposition in the case $A=D_{r_{0}}\left(G^{p^{m}}\right)$. By Lem. 5.15] and the discussion above the ring $A$ is seen to be Auslander regular of global dimension $\leq d$ and, at the same time, a complete doubly filtered $K$-algebra with $\operatorname{Gr}(A)=k\left[u_{1}, \ldots, u_{d}\right]$. Furthermore, functoriality of $D(\cdot)$ gives a $K$-algebra homomorphism

$$
D\left(G^{p^{m}}\right) \rightarrow D(\{1\})=K
$$

which extends to $A$ and hence, $A$ admits a nonzero module of finite $K$-dimension. Now [4], Prop. 9.1 completes the proof.

For future reference we repeat the following ingredient of the preceding proof.

Corollary 9.4. If $M$ denotes a finitely generated $D_{r_{m}}(G)$-module $M$ then

$$
d_{D_{r_{m}}(G)}(M)=d_{D_{r_{0}}\left(G^{p^{m}}\right)}(M) \text {. }
$$

9.5. As before we now let $\mathbf{G}$ be a connected split reductive group scheme over $o_{L}$ with Lie algebra $\mathfrak{g}$. We assume that (H1)-(H3) from (4.10) hold. Let $\mathbf{G}_{\mathbb{C}}^{\prime}$ denote the complex derived algebraic group of $\mathbf{G}$ and let $\mathfrak{g}_{\mathbb{C}}^{\prime}$ be its Lie algebra. It is known that there is a unique non-zero coadjoint $\mathbf{G}_{\mathbb{C}^{-}}^{\prime}$ orbit in $\left(\mathfrak{g}_{\mathbb{C}}^{\prime}\right)^{*}$ of minimal dimension ([14], Rem. 4.3.4) and its dimension is an even integer $\geq 2$. Let $r$ denote half this dimension. According to work of A. Joseph ([28]) the values of $r$ are well-known. The following table is taken from loc.cit. with the two corrections noted in [29].

\begin{tabular}{c|ccccccccc}
$\Phi$ & $A_{l}$ & $B_{l}$ & $C_{l}$ & $D_{l}$ & $E_{6}$ & $E_{7}$ & $E_{8}$ & $F_{4}$ & $G_{2}$ \\
\hline $\operatorname{dim} \mathfrak{g}_{\mathbb{C}}^{\prime}$ & $l^{2}+2 l$ & $2 l^{2}+l$ & $2 l^{2}+l$ & $2 l^{2}-l$ & 78 & 133 & 248 & 52 & 14 \\
$r$ & $l$ & $2 l-2$ & $l$ & $2 l-3$ & 11 & 17 & 29 & 8 & 3.
\end{tabular}


9.6. Let $\tilde{G}$ be from now on a locally $L$-analytic group whose $L$-Lie algebra $\operatorname{Lie}(\tilde{G})$ is isomorphic to $\mathfrak{g}_{L}:=L \otimes_{o_{L}} \mathfrak{g}$. Let us identify these Lie algebras via such an isomorphism. Let $d=\operatorname{dim}_{L} \operatorname{Lie}(\tilde{G})$. Choose $k \geq 1$ sufficiently large such that $\exp _{\tilde{G}}$ is defined on $\Lambda:=\pi_{L}^{k} \mathfrak{g}$ and such that

$$
G:=\exp _{\tilde{G}}(\Lambda)
$$

is an open subgroup of $\tilde{G}$ which is $L$-uniform. Then

$$
L_{G}=\Lambda=\pi_{L}^{k} \mathfrak{g}_{0}
$$

as Lie algebras over $\mathbb{Z}_{p}$ where $\mathfrak{g}_{0}$ equals $\mathfrak{g}$ but viewed over $\mathbb{Z}_{p}$. In other words, the condition (HYP) from 6.6 is satisfied. Let $m \geq 1$. According to Prop. 6.10 we have a canonical algebra isomorphism

$$
\mathcal{L}_{G^{p}}: \widehat{U(\mathfrak{g})_{n}, K} \stackrel{\cong}{\cong} D_{r_{0}}\left(G^{p^{m}}, K\right)
$$

where $n:=(m-1) e+k \geq 1$. At this point we recall a deep theorem of [4].

Theorem 9.7. (Ardakov/Wadsley) Let $A:=\widehat{U(\mathfrak{g})_{n}, K}$. If $M$ is a finitely generated (left) A-module with $d_{A}(M) \geq 1$, then $d_{A}(M) \geq r$.

Proof. It is straightforward to check that the results of loc.cit., 9.3/4/5/6 extend from the semisimple case considered there to the case of $\mathbf{G}$. We may therefore assume, by passing to a finite field extension of $K$, that $M$ is a (finitely generated) $\widehat{\mathcal{U}_{n}^{\lambda}, K^{\lambda}}$-module for a weight $\lambda \in \mathfrak{t}_{K}^{*}$ such that $\lambda+\rho$ is dominant. There is a canonical decomposition as Lie algebras $\mathfrak{g}=\mathfrak{a} \times \mathfrak{g}^{\prime}$ with abelian $\mathfrak{a}$ and a semisimple algebra $\mathfrak{g}^{\prime} \neq 0$. The (completed deformed) central reduction $\widehat{\mathcal{U}_{n}^{\lambda}}$ depends only on $\mathfrak{g}^{\prime}$. The claim follows now as in the proof of loc.cit., Thm. 9.10.

Remark: Thm. 9.10 of loc.cit. is the analogue of Smith's theorem for $p$-adically completed universal enveloping algebras. It allows to prove a similar analogue for certain Iwasawa algebras culminating in the main result of loc.cit.

By Cor. 9.4 we have

Corollary 9.8. If $M$ is a finitely generated (left) $D_{r_{m}}(G)$-module with $d_{D_{r_{m}}(G)}(M) \geq 1$ then $d_{D_{r_{m}}(G)}(M) \geq r$.

As we have pointed out in section 5 the projective system of noetherian $K$-Banach algebras $D_{r_{m}}(G)$ for $m \geq 1$ defines the Fréchet-Stein structure of $D(G)$. By Prop. 9.3 the rings $D_{r_{m}}(G)$ are Auslander regular with global dimension equal to $d$. This implies ([54], §8) that the category of coadmissible modules $\mathscr{C}_{\tilde{G}}$ is equipped with a well-behaved canonical codimension function defined as $\operatorname{codim}(M):=j_{D(G)}(M)$ for a coadmissible module $M$. This number does not depend on the choice of $G$. Let $\operatorname{dim}(M):=d-\operatorname{codim} M$. Hence $0 \leq \operatorname{dim}(M) \leq d$ if $M \neq 0$ and $\operatorname{dim}(0)=-\infty$.

Theorem 9.9. Let $M \in \mathscr{C}_{\tilde{G}}$ with $\operatorname{dim}(M) \geq 1$. Then $\operatorname{dim}(M) \geq r$. 
Proof. According to [54], Lem. 8.4 we have $\operatorname{dim}(M):=\sup _{m \geq 1} d_{D_{r_{m}}(G)}\left(M_{m}\right)$ and hence $\operatorname{dim}(M)=d_{D_{r_{m^{\prime}}}(G)}\left(M_{m^{\prime}}\right) \geq 1$ for a particular $m^{\prime} \geq 1$. Now apply Cor. 9.8 to $M_{m^{\prime}}$.

Proposition 9.10. Let $M \in \mathscr{C}_{\tilde{G}}$. Then $M$ is zero-dimensional if and only if the coherent sheaf of $M$ consists of finite-dimensional $K$-vector spaces.

Proof. If $M$ is zero-dimensional then $d_{D_{r_{m}}(G)}\left(M_{m}\right)=0$ for all $m \geq 1$ and the claim follows from Prop. 9.3. The other implication is [46], Thm. 8.4.

\section{REFERENCES}

[1] Y. Amice. Duals. In Proceedings of the Conference on p-adic Analysis (Nijmegen, 1978), volume 7806 of Report, pages 1-15. Katholieke Univ. Nijmegen, 1978.

[2] K Ardakov. Krull dimension of Iwasawa algebras and some related topics. PhD-thesis, Cambridge, UK (2004), available online at: http://www.maths.nottingham.ac.uk/personal/pmzka1/.

[3] K. Ardakov and S. Wadsley. On the Cartan map for crossed products and Hopf-Galois extensions. Algebras and Representation Theory, 13(1):33-41, 2010.

[4] K. Ardakov and S. Wadsley. On irreducible representations of compact $p$-adic analytic groups. To appear in: The Annals of Mathematics, available online at: http://arxiv.org/abs/1102.2606.

[5] A. Beulinson and J. Bernstein. Localisation de g-modules. C. R. Acad. Sci. Paris Sér. I Math., 292(1):15-18, 1981.

[6] Alexander Beilinson and Victor Ginzburg. Wall-crossing functors and $\mathcal{D}$-modules. Represent. Theory, 3:1-31, 1999.

[7] P. Berthelot. D-modules arithmétiques I. Opérateurs différentiels de niveau fini. Ann. Sci. E.N.S, 29:185-272, 1996.

[8] Pierre Berthelot and Arthur Ogus. Notes on crystalline cohomology. Princeton University Press, Princeton, N.J., 1978.

[9] Mirković I. Rumynin D. Bezrukavnikov, R. Localisation of modules for a semisimple Lie algebra in prime characteristic. Ann. of Math., 167(3):945-991, 2008.

[10] Walter Borho and Jean-Luc Brylinski. Differential operators on homogeneous spaces. II. Relative enveloping algebras. Bull. Soc. Math. France, 117(2):167-210, 1989.

[11] S. Bosch, U. Güntzer, and R. Remmert. Non-Archimedean analysis. Springer-Verlag, Berlin, 1984.

[12] N. Bourbaki. Éléments de mathématique. Fasc. XXXIII. Variétés différentielles et analytiques. Fascicule de résultats (Paragraphes 1 à 7). Actualités Scientifiques et Industrielles, No. 1333. Hermann, Paris, 1967.

[13] N. Bourbaki. Éléments de mathématique. Fasc. XXXVII. Groupes et algèbres de Lie. Chap. II/III. Hermann, Paris, 1972. Act.Sci. et Ind., No. 1349.

[14] David H. Collingwood and William M. McGovern. Nilpotent orbits in semisimple Lie algebras. Van Nostrand Reinhold Mathematics Series. New York, 1993.

[15] M. Demazure. Invariants symétriques entiers des groupes de Weyl et torsion. Invent. Math., 21:287301, 1973.

[16] Michel Demazure and Pierre Gabriel. Groupes algébriques. Tome I: Géométrie algébrique, généralités, groupes commutatifs. Masson \& Cie, Éditeur, Paris, 1970. Avec un appendice it Corps de classes local par Michiel Hazewinkel.

[17] Jacques Dixmier. Enveloping algebras, volume 11 of Graduate Studies in Mathematics. American Mathematical Society, Providence, RI, 1996. Revised reprint of the 1977 translation.

[18] J. D. Dixon, M. P. F. du Sautoy, A. Mann, and D. Segal. Analytic pro-p groups, volume 61 of Cambridge Studies in Advanced Mathematics. Cambridge University Press, Cambridge, second edition, 1999. 
[19] G. Dospinescu and B. Schraen. Endomorphism algebras of admissible $p$-adic representations of $p$-adic Lie groups. To appear in: Representation Theory.

[20] M. Emerton. On the interpolation of systems of eigenvalues attached to automorphic Hecke eigenforms. Invent. Math., 164(1):1-84, 2006.

[21] A. Grothendieck. Éléments de géométrie algébrique. I. Le langage des schémas. Inst. Hautes Études Sci. Publ. Math., (4):228, 1960.

[22] A. Grothendieck. Éléments de géométrie algébrique. IV. Étude locale des schémas et des morphismes de schémas. III. Inst. Hautes Études Sci. Publ. Math., (28):255, 1966.

[23] A. Grothendieck. Éléments de géométrie algébrique. IV. Étude locale des schémas et des morphismes de schémas IV. Inst. Hautes Études Sci. Publ. Math., (32):361, 1967.

[24] Annette Huber, Guido Kings, and Niko Naumann. Some complements to the Lazard isomorphism. Compos. Math., 147(1):235-262, 2011.

[25] Birger Iversen. Cohomology of sheaves. Universitext. Springer-Verlag, Berlin, 1986.

[26] Jens Carsten Jantzen. Representations of Lie algebras in prime characteristic. In Representation theories and algebraic geometry (Montreal, PQ, 1997), volume 514 of NATO Adv. Sci. Inst. Ser. C Math. Phys. Sci., pages 185-235. Kluwer Acad. Publ., Dordrecht, 1998. Notes by Iain Gordon.

[27] Jens Carsten Jantzen. Representations of algebraic groups, volume 107 of Mathematical Surveys and Monographs. American Mathematical Society, Providence, RI, second edition, 2003.

[28] A. Joseph. Minimal realizations and spectrum generating algebras. Comm. Math. Phys., 36:325-328, 1974.

[29] A. Joseph. The minimal orbit in a simple lie algebra and its associated maximal ideal. Ann. Scient. Ec. Norm Sup., 9:1-30, 1976.

[30] R. Kiehl. Theorem A und Theorem B in der nichtarchimedischen Funktionentheorie. Invent. Math., 2:256-273, 1967.

[31] J. Kohlhaase. Invariant distributions on p-adic analytic groups. Duke Math. J., 137(1):19-62, 2007.

[32] M. Lazard. Groupes analytiques p-adiques. Inst. Hautes Études Sci. Publ. Math., (26):389-603, 1965.

[33] M. Lazard. Groupes analytiques p-adiques. Inst. Hautes Études Sci. Publ. Math., (26):4-219, 1965.

[34] Thierry Levasseur. Some properties of noncommutative regular graded rings. Glasgow Math. J., 34(3):277-300, 1992.

[35] H. Li and F. van Oystaeyen. Zariskian filtrations, volume 2 of $K$-Monographs in Mathematics. Kluwer Academic Publishers, Dordrecht, 1996.

[36] D. Loeffler. Overconvergent algebraic automorphic forms. Proc. London Math. Soc. Oxford Math. J., 102(2):193-228, 2011.

[37] J. C. McConnell and J. C. Robson. Noncommutative Noetherian rings. Pure and Applied Mathematics (New York). John Wiley \& Sons Ltd., Chichester, 1987.

[38] D. Miličić. Algebraic D-modules and representation theory of semisimple Lie groups. In Analytic Cohomology and Penrose Transform, volume 154 of Contemporary Mathematics, pages 133-168. Amer. Math. Soc., Providence, RI, 1993.

[39] D. Miličić. Localization and Representation Theory of Reductive Lie groups. Preprint 1993, available online at: http://www.math.utah.edu/ milicic.

[40] J. S. Milne. Étale cohomology, volume 33 of Princeton Math. Series. Princeton Univ. Press, Princeton, N.J., 1980.

[41] C. Noot-Huyghe. Un théorème de Beilinson-Bernstein pour les D-modules arithmétiques. Bull. Soc. Math. France, 137(2):159-183, 2009.

[42] S. Orlik and M. Strauch. On the irreducibility of locally analytic principal series representations. Represent. Theory, 14:713-746, 2010.

[43] D.S. Passman. Infinite crossed products, volume 135 of Pure and Applied Mathematics. Academic Press Inc., Boston, MA, 1989. 
[44] D. Patel, T. Schmidt, and M. Strauch. Locally analytic representations and sheaves on the Bruhat-Tits building. Preprint 2012, available online at: http://www.math.unimuenster.de/u/tobias.schmidt.

[45] T. Schmidt. Verma modules over $p$-adic Arens-Michael envelopes of reductive Lie algebras. Preprint 2010, submitted to: Journal of Algebra.

[46] T. Schmidt. Auslander regularity of $p$-adic distribution algebras. Represent. Theory, 12:37-57, 2008.

[47] T. Schmidt. Analytic vectors in continuous p-adic representations. Compositio Math., 145:247-270, 2009.

[48] T. Schmidt. Stable flatness of nonarchimedean hyperenveloping algebras. Journal of Algebra, 323/3:757-765, 2010.

[49] P. Schneider. Nonarchimedean functional analysis. Springer Monographs in Mathematics. SpringerVerlag, Berlin, 2002.

[50] P. Schneider. Continuous representation theory of $p$-adic Lie groups. In International Congress of Mathematicians. Vol. II, pages 1261-1282. Eur. Math. Soc., Zürich, 2006.

[51] P. Schneider and J. Teitelbaum. U(g)-finite locally analytic representations. Represent. Theory, 5:111-128 (electronic), 2001. With an appendix by Dipendra Prasad.

[52] P. Schneider and J. Teitelbaum. Locally analytic distributions and $p$-adic representation theory, with applications to $\mathrm{GL}_{2}$. J. Amer. Math. Soc., 15(2):443-468 (electronic), 2002.

[53] P. Schneider and J. Teitelbaum. p-adic boundary values. Astérisque, (278):51-125, 2002. Cohomologies $p$-adiques et applications arithmétiques, I.

[54] P. Schneider and J. Teitelbaum. Algebras of $p$-adic distributions and admissible representations. Invent. Math., 153(1):145-196, 2003.

[55] P. Schneider and J. Teitelbaum. Duality for admissible locally analytic representations. Represent. Theory, 9:297-326 (electronic), 2005.

[56] S.P. Smith. Krull dimension of factor rings of the enveloping algebra of a semisimple Lie algebra. Math. Proc. Cambridge Philos. Soc., 93(3):459-466, 1983.

Mathematisches Institut, Westfä̈ische Wilhelms-Universität Münster, Einsteinstr. 62, D-48149 Münster, Germany

E-mail address: toschmid@math.uni-muenster.de 